\title{
DZ Chamaeleontis: a bona fide photoevaporating disc ${ }^{\star}$
}

\author{
H. Canovas ${ }^{1,2}$, B. Montesinos ${ }^{3}$, M. R. Schreiber ${ }^{4,11,12}$, L. A. Cieza ${ }^{5,11}$, C. Eiroa ${ }^{1}$, G. Meeus ${ }^{1}$, J. de Boer ${ }^{6}$, F. Ménard ${ }^{7}$,
} Z. Wahhaj ${ }^{8}$, P. Riviere-Marichalar ${ }^{9}$, J. Olofsson ${ }^{4,12}$, A. Garufi ${ }^{1}$, I. Rebollido ${ }^{1}$, R. G. van Holstein ${ }^{6}$, C. Caceres ${ }^{10,11,12}$, A. Hardy ${ }^{4,11}$, and E. Villaver ${ }^{1}$

1 Departamento de Física Teórica, Universidad Autónoma de Madrid, Cantoblanco, 28049 Madrid, Spain e-mail: hcanovas@sciops.esa.int

2 European Space Astronomy Centre (ESA), Camino Bajo del Castillo s/n, 28692 Villanueva de la Cañada, Madrid, Spain

3 Dept. of Astrophysics, Centre for Astrobiology (CAB, CSIC-INTA), ESAC Campus, Camino Bajo del Castillo s/n, 28692 Villanueva de la Cañada, Madrid, Spain

4 Departamento de Física y Astronomía, Universidad de Valparaíso, Casillo 5030, Valparaíso, Chile

5 Facultad de Ingeniería y Ciencias, Universidad Diego Portales, Av. Ejercito 441, Santiago, Chile

${ }^{6}$ Leiden Observatory, Leiden University, PO Box 9513, 2300 RA Leiden, The Netherlands

7 Univ. Grenoble Alpes, CNRS, IPAG, 38000 Grenoble, France

8 European Southern Observatory, 3107 Alonso de Cordova, Vitacura, 1058 Santiago, Chile

9 Instituto de Ciencia de Materiales de Madrid (ICMM-CSIC), 28049 Cantoblanco, Madrid, Spain

10 Departamento de Ciencias Fisicas, Facultad de Ciencias Exactas, Universidad Andres Bello Av. Fernandez Concha 700, Las Condes, Santiago, Chile

11 Millennium Nucleus, Protoplanetary discs in ALMA Early Science, 1058 Santiago, Chile

12 Millennium Nucleus for Planet Formation, Chile

Received 25 July 2017 / Accepted 9 October 2017

\section{ABSTRACT}

\begin{abstract}
Context. DZ Cha is a weak-lined T Tauri star (WTTS) surrounded by a bright protoplanetary disc with evidence of inner disc clearing. Its narrow $\mathrm{H} \alpha$ line and infrared spectral energy distribution suggest that DZ Cha may be a photoevaporating disc.

Aims. We aim to analyse the DZ Cha star + disc system to identify the mechanism driving the evolution of this object.

Methods. We have analysed three epochs of high resolution optical spectroscopy, photometry from the UV up to the sub-mm regime, infrared spectroscopy, and $J$-band imaging polarimetry observations of DZ Cha.

Results. Combining our analysis with previous studies we find no signatures of accretion in the $\mathrm{H} \alpha$ line profile in nine epochs covering a time baseline of $\sim 20 \mathrm{yr}$. The optical spectra are dominated by chromospheric emission lines, but they also show emission from the forbidden lines [SII] 4068 and [OI] $6300 \AA$ that indicate a disc outflow. The polarized images reveal a dust depleted cavity of $\sim 7$ au in radius and two spiral-like features, and we derive a disc dust mass limit of $M_{\text {dust }}<3 M_{\text {Earth }}$ from the sub-mm photometry. No stellar $\left(M_{\star}>80 M_{\text {Jup }}\right)$ companions are detected down to 0 ”.07 ( $\sim 8$ au, projected).

Conclusions. The negligible accretion rate, small cavity, and forbidden line emission strongly suggests that DZ Cha is currently at the initial stages of disc clearing by photoevaporation. At this point the inner disc has drained and the inner wall of the truncated outer disc is directly exposed to the stellar radiation. We argue that other mechanisms like planet formation or binarity cannot explain the observed properties of DZ Cha. The scarcity of objects like this one is in line with the dispersal timescale $\left(\lesssim 10^{5} \mathrm{yr}\right)$ predicted by this theory. DZ Cha is therefore an ideal target to study the initial stages of photoevaporation.
\end{abstract}

Key words. accretion, accretion disks - protoplanetary disks - stars: variables: T Tauri, Herbig Ae/Be

\section{Introduction}

T Tauri stars (TTS) are pre-main sequence stars (PMS) of late spectral types (SpT, F-M) and typical masses $<1.5 M_{\odot}$ (e.g. Bertout 1989). They are surrounded by circumstellar discs created during the collapse of the protostellar cores. Since their discovery by Joy (1945), TTS have attracted wide attention from the astronomy community, and nowadays it is accepted that planets can form in their surrounding discs (Sallum et al. 2015; Quanz et al. 2015). Understanding how these discs evolve is therefore key to constraining theories of planet formation and eventually to explaining the diversity of currently observed planetary architectures (e.g. Winn \& Fabrycky 2015, and references therein).

\footnotetext{
* Based on observations collected at the European Organisation for Astronomical Research in the Southern Hemisphere under ESO programme 097.C-0536. Based on data obtained from the ESO Science Archive Facility under request number 250112.
}

Historically, TTS have been classified into two groups according to their accretion tracers (mainly the $\mathrm{H} \alpha$ line equivalent width, $E W(\mathrm{H} \alpha)$ ). Classical T Tauri stars (CTTS) show broad $\mathrm{H} \alpha$ line profiles (usually $E W(\mathrm{H} \alpha) \geq 10 \AA$ ), strong emission lines at ultraviolet (UV) and optical wavelengths, and/or veiling signatures across the UV and optical ranges. These observables indicate active accretion of material onto the star, with mass accretion rates ranging from $10^{-7}-10^{-9} M_{\odot} \mathrm{yr}^{-1}$ (Gullbring et al. 1998). Thermal emission from their dusty circumstellar discs is usually detected above photospheric levels in nearly the entire infrared and sub-mm regime (e.g. Evans et al. 2009; Howard et al. 2013; Ansdell et al. 2016). On the other hand, Weak-lined T Tauri stars (WTTS) show no evidence of accretion activity. Their spectra show no veiling signatures, and they lack most of the emission lines observed in CTTS. Their $\mathrm{H} \alpha$ line is mostly dominated by chromospheric emission and is typically narrow $(E W(\mathrm{H} \alpha)<10 \AA ̊)$. Hot circumstellar dust, probed at near- and mid-infrared wavelengths, is detected in only $20 \%$ 
of them (Padgett et al. 2006; Cieza et al. 2007; Wahhaj et al. 2010), and this excess is systematically fainter than in CTTS. In the sub-mm regime their discs are usually fainter than the more luminous discs around CTTS (Andrews \& Williams 2005), and some of them have disc masses comparable to the gas-poor debris discs (Hardy et al. 2015). Both CTTS and WTTS are bright X-ray sources, and in fact WTTS (originally called naked TTS) were first discovered via X-rays surveys (Walter et al. 1988).

As they evolve, all CTTS will become WTTS before entering the main sequence (MS). In other words, the discs around CTTS will eventually be very depleted of gas and dust, and accretion onto the star will be too faint to leave a detectable imprint in the stellar spectrum. Discs showing evidence of inner cavities and/or gaps are thought to be in transition between these two evolutionary stages (Strom et al. 1989). The scarcity of these objects indicates that this transition phase lasts $\lesssim 0.5 \mathrm{Myr}$ (e.g. Wolk \& Walter 1996; Cieza et al. 2007), and multiwavelength observations indicate that once the process begins the entire disc quickly dissipates from inside out (Duvert et al. 2000). Photoevaporation from the central star in combination with viscous accretion is, to date, the most successful theory in explaining this rapid transition (Alexander et al. 2014). Current observational evidence supports this theory; several discs show photoevaporative winds traced by the [O I] $(6300 \AA)$ and [Ne II] $(12.8 \mu \mathrm{m})$ forbidden emission lines and by free-free emission at centimetre wavelengths (Pascucci \& Sterzik 2009; Sacco et al. 2012; Rigliaco et al. 2013; Macías et al. 2016). Photoevaporation models show that beyond a critical radius (a few au for $1 M_{\odot}$ stars), the stellar radiation creates a pressure gradient in the disc upper layers that drives a photoevaporative wind (Hollenbach et al. 1994; Font et al. 2004). This process strongly depends on the energy regime dominating the photoevaporation (far-ultraviolet, FUV; extreme ultraviolet, EUV; and/or $\mathrm{X}$-rays), with predicted photoevaporation rates ranging from $10^{-8}-10^{-10} M_{\odot} \mathrm{yr}^{-1}$ (Ercolano et al. 2009; Gorti \& Hollenbach 2009; Owen et al. 2012). These models qualitatively agree on the initial steps of disc clearing: once the stellar mass accretion rate drops to the level of the photoevaporation mass loss rates, the flow of material from the outer disc towards the star is interrupted at the critical radius. When this happens the inner disc (the region encompassed by the critical radius) is detached from the outer disc and it is quickly accreted by the star (Clarke et al. 2001). At this point, the inner edge of the outer disc is directly exposed to the stellar radiation and the entire outer disc disperses from the inside out in $\sim 10^{5}$ yr (Alexander et al. 2006a,b). Despite the success in explaining the evolution of protoplanetary discs, observations confirming the first steps of the photoevaporation predicted by the theory are still missing.

DZ Cha (alias PDS 59; RX J1149.8-7850, $\alpha=$ $\left.11^{\mathrm{h}} 49^{\mathrm{m}} 31.84^{\mathrm{s}}, \delta=-78^{\circ} 51^{\prime} 01.1^{\prime \prime}\right)$ is a peculiar M0Ve TTs (Gregorio-Hetem et al. 1992; Torres et al. 2006). Although studies link it to the nearby $\beta$ Pic association (Riaz et al. 2006; Malo et al. 2013, 2014), given its proper motions and Li $6708 \AA$ equivalent width DZ Cha most likely belongs to the more distant $\epsilon$ Cha sparse stellar association (Torres et al. 2006, 2008; Luhman et al. 2008; Lopez Martí et al. 2013; Murphy et al. 2013; Elliott et al. 2014). In a detailed study of the $\epsilon$ Cha association, Murphy et al. (2013) derive a distance of $d=110 \pm 7 \mathrm{pc}$ and an age of 2-3 Myr for DZ Cha. This star shows strong flaring events (Guenther \& Emerson 1997; Tripicchio et al. 2000), and was first classified as a WTTS by Alcala et al. (1995). DZ Cha has a very narrow $\mathrm{H} \alpha$ line profile, with a full width at $10 \%$ of the line of FW0.1 $(\mathrm{H} \alpha) \sim 120 \mathrm{~km} \mathrm{~s}^{-1}$ and equivalent
Table 1. UVES observation log.

\begin{tabular}{cccc}
\hline \hline $\begin{array}{c}\text { Date } \\
(\text { yy-mm-dd) }\end{array}$ & $\begin{array}{c}\langle\text { Seeing }\rangle \\
\left({ }^{\prime \prime}\right)\end{array}$ & $\langle$ Airmass $\rangle$ & $\begin{array}{c}\text { Int. time } \\
(\mathrm{s})\end{array}$ \\
\hline $2012-01-09$ & 0.80 & 1.77 & 575 \\
$2012-02-24$ & 0.94 & 1.72 & 575 \\
$2012-03-07$ & 0.91 & 1.74 & 575
\end{tabular}

Notes. The columns list the observation date, average airmass, seeing, and total integration time.

width $E W(\mathrm{H} \alpha)<5 \AA$ (Torres et al. 2006; Wahhaj et al. 2010). Its near-infrared (NIR) SED shows evidence of an inner cavity, but at longer wavelengths its strong excess is virtually indistinguishable from the SEDs of accreting discs around CTTS (Luhman et al. 2008; Wahhaj et al. 2010). In fact, its fractional disc luminosity is the highest among the hundreds of WTTS observed during the Spitzer "c2d" program (Wahhaj et al. 2010).

Given the properties of DZ Cha we selected this object as an ideal target to study the transition from CTTS to WTTS. In this paper we present the first detailed analysis of this interesting disc. We show that the evidence of inner disc clearing in combination with its negligible accretion rate across different epochs, strong disc emission at mid-infrared wavelengths, and the disc outflow probed by forbidden emission lines, are best explained if DZ Cha is at the initial stages of inner disc clearing by photoevaporation. Our observations and results are presented in Sects. 2 and 3, respectively. The results are discussed in Sect. 4, and a summary and conclusions are presented in Sect. 5.

\section{Observations and data reduction}

We have combined three epochs of high resolution optical spectroscopy, photometry ranging from the UV to the sub-mm regime, and imaging polarimetry at $J$ band. Below we describe the observations and data processing.

\subsection{Optical spectroscopy}

High resolution (processed) spectroscopy at optical wavelengths was retrieved from the ESO Science Archive facility. The observations were acquired with the Ultraviolet and Visual Echelle Spectrograph (UVES) at the Very Large Telescopes (VLT's) as part of the ESO program ID 088.C-0506 (PI: C. Torres) over three nights. A summary of the observations is given in Table 1. The instrument was configured using the 1'”0 slit that samples the $3250-6800 \AA$ range with a resolution of $\lambda / \Delta \lambda \sim 40000$. Each of the three datasets has a signal-to-noise ratio $(\mathrm{S} / \mathrm{N})$ of $\sim 80$ in the continuum around the Li $6708 \AA$ line. Barycentric and stellar radial velocity (Elliott et al. 2014) corrections were applied with customized scripts.

\subsection{Photometry}

In this work we use calibrated photometry publicly available in the Vizier Service ${ }^{1}$ and at the NASA/IPAC Infrared Science Archive (IRSA) ${ }^{2}$. Ultraviolet (UV) photometry was obtained from the GALEX catalogue of UV sources (Bianchi et al. 2011). Optical photometry (Johnson $B$ and $V$, and Sloan $g^{\prime}$, $r^{\prime}$, and $i^{\prime}$ ) was obtained from the American Association of Variable Star Observers (AAVSO) Photometric All Sky Survey

\footnotetext{
http://vizier.u-strasbg.fr/viz-bin/VizieR

http://irsa.ipac.caltech.edu/frontpage/
} 
(APASS, Henden et al. 2016). Infrared photometry was obtained from the Two Micron All Sky Survey (2MASS, Skrutskie et al. 2006), from the Spitzer c2d legacy program (Evans et al. 2003), from the ALLWISE catalogue (Cutri et al. 2014), and from the Herschel/SPIRE point source catalogue. We also obtained the Spitzer/IRS spectra acquired during the Spitzer GO program \#50053 (PI: Houck, J. R.). Visual inspection of these catalogues shows no companions and/or background emission that may contaminate the published photometry and IRS spectra.

Furthermore, we retrieved raw Herschel/PACS (70 and $160 \mu \mathrm{m})$ observations from the Herschel Science Archive ${ }^{3}$ and processed them following Riviere-Marichalar et al. (2013). The Herschel Interactive Processing Environment (HIPE) 14 with the most recent version for the calibration files was used to identify and remove bad pixels, to apply flat field correction, and for deglitching, high pass filtering, and map projection. Aperture photometry was measured in the processed images using an aperture of $6^{\prime \prime}$ and $12^{\prime \prime}$ for the 70 and $160 \mu \mathrm{m}$ images, respectively. The sky was measured in a ring centred on the star with radius and width of $25^{\prime \prime}$ and $10^{\prime \prime}$, respectively. The final uncertainties in the photometry were computed as the quadratic sum of the photometry and calibration errors.

Sub-mm photometry was obtained with the Atacama Pathfinder Experiment $\left(\mathrm{APEX}^{4}\right)$, the $12 \mathrm{~m}$ radio telescope located on Llano de Chajnantor (Chile). Our observations were performed during period 092 (C-092.F-9701A-2013). We used the APEX-LABOCA camera (Siringo et al. 2009) which operates at a central frequency of $345 \mathrm{GHz}(870 \mu \mathrm{m})$ aiming to detect the dust continuum emission from DZ Cha. The APEX pointing uncertainty is $2^{\prime \prime}$ (Güsten et al. 2006), and the nominal LABOCA beam full width at half maximum (FWHM) is $19.2 \pm 0.7^{\prime \prime}$. The most sensitive part of the bolometer array was centred on the target to maximize the chance of detection. Skydips and radiometer measurements were interleaved during the observation to obtain accurate atmospheric opacity estimates. Mars and the quasar PKS1057-79 were used to calibrate the focus and pointing, respectively. The secondary calibrator NGC 2071 was used to perform the absolute flux calibration, which is expected to be accurate to within $10 \%$ (Siringo et al. 2009). The emission around DZ Cha was mapped following a raster map in spiral mode. The weather conditions were excellent with a nearly constant precipitable water vapour (PWV) of $0.22 \mathrm{~mm}$. A total of ten scans were obtained, resulting in $1.52 \mathrm{~h}$ of on-source integration time. The observations were reduced with the CRUSH package (Kovács 2008) using the faint reduction mode and half a beam smoothing factor. This way we reached an rms of $8 \mathrm{mJy}_{\text {beam }}^{-1}$, but no emission was detected at the location of DZ Cha.

The observed and de-reddened fluxes (see Sect. 3.1.1) are listed in Table 2.

\subsection{Imaging polarimetry}

We observed DZ Cha with the SPHERE (Spectro-Polarimetric High-contrast Exoplanet REsearch, Beuzit et al. 2008) instrument at the VLT on April 4, 2016, under ESO programme 097.C-0536(A). Observations were carried out with the infrared subsystem IRDIS (Infra-Red Dual Imaging and Spectrograph, Dohlen et al. 2008) in the dual polarization imaging mode (DPI,

\footnotetext{
3 http://archives.esac.esa.int/hsa/whsa/

4 This publication is based on data acquired with APEX which is a collaboration between the Max-Planck-Institut fur Radioastronomie, the European Southern Observatory, and the Onsala Space Observatory.
}

Table 2. SED of DZ Cha.

\begin{tabular}{ccccc}
\hline $\begin{array}{c}\text { Wavelength } \\
(\mu \mathrm{m})\end{array}$ & $\begin{array}{c}\text { Flux } \\
(\mathrm{mJy})\end{array}$ & $\begin{array}{c}\text { Flux Dered. } \\
(\mathrm{mJy})\end{array}$ & $\begin{array}{c}\text { Error } \\
(\%)\end{array}$ & Ref. \\
\hline 0.15 & $2.20 \mathrm{E}-02$ & $5.46 \mathrm{E}-02$ & 30 & 1 \\
0.23 & $8.50 \mathrm{E}-02$ & $2.26 \mathrm{E}-01$ & 30 & 1 \\
0.44 & $7.90 \mathrm{E}+00$ & $1.25 \mathrm{E}+01$ & 15 & 2 \\
0.48 & $1.38 \mathrm{E}+01$ & $2.10 \mathrm{E}+01$ & 15 & 2 \\
0.55 & $2.85 \mathrm{E}+01$ & $4.01 \mathrm{E}+01$ & 10 & 2 \\
0.62 & $5.09 \mathrm{E}+01$ & $6.79 \mathrm{E}+01$ & 10 & 2 \\
0.76 & $1.17 \mathrm{E}+02$ & $1.45 \mathrm{E}+02$ & 10 & 2 \\
1.24 & $2.62 \mathrm{E}+02$ & $2.90 \mathrm{E}+02$ & 5 & 3 \\
1.65 & $3.41 \mathrm{E}+02$ & $3.63 \mathrm{E}+02$ & 5 & 3 \\
2.16 & $2.72 \mathrm{E}+02$ & $2.83 \mathrm{E}+02$ & 5 & 3 \\
3.35 & $1.66 \mathrm{E}+02$ & $1.69 \mathrm{E}+02$ & 5 & 4 \\
3.55 & $1.65 \mathrm{E}+02$ & $1.69 \mathrm{E}+02$ & 5 & 5 \\
4.49 & $1.73 \mathrm{E}+02$ & $1.76 \mathrm{E}+02$ & 5 & 5 \\
4.60 & $1.54 \mathrm{E}+02$ & $1.57 \mathrm{E}+02$ & 5 & 4 \\
5.73 & $1.90 \mathrm{E}+02$ & $1.93 \mathrm{E}+02$ & 5 & 5 \\
7.87 & $3.66 \mathrm{E}+02$ & $3.72 \mathrm{E}+02$ & 5 & 5 \\
11.56 & $4.42 \mathrm{E}+02$ & $4.44 \mathrm{E}+02$ & 5 & 4 \\
22.09 & $1.54 \mathrm{E}+03$ & $1.55 \mathrm{E}+03$ & 5 & 4 \\
23.67 & $1.24 \mathrm{E}+03$ & $1.24 \mathrm{E}+03$ & 10 & 5 \\
70.00 & $1.73 \mathrm{E}+03$ & $1.73 \mathrm{E}+03$ & 5 & 6 \\
160.00 & $1.09 \mathrm{E}+03$ & $1.09 \mathrm{E}+03$ & 5 & 6 \\
250.00 & $3.48 \mathrm{E}+02$ & $3.48 \mathrm{E}+02$ & 5 & 6 \\
363.00 & $1.59 \mathrm{E}+02$ & $1.59 \mathrm{E}+02$ & 5 & 6 \\
517.00 & $7.41 \mathrm{E}+01$ & $7.41 \mathrm{E}+01$ & 15 & 6 \\
870.00 & $<2.40 \mathrm{E}+01^{a}$ & $<2.40 \mathrm{E}+01^{a}$ & $\ldots$ & 6 \\
\hline & & & & \\
\hline
\end{tabular}

Notes. The columns list the effective wavelength of each filter, the observed flux, the de-reddened flux, and the associated errors. Extinction uncertainties dominate at wavelengths shorter than $1 \mu \mathrm{m}$, while calibration uncertainties dominate at longer wavelengths. ${ }^{(a)} 3 \sigma$ upper limit. Photometry references: 1) Bianchi et al. (2011); 2) Henden et al. (2016); 3) Cutri et al. (2003); 4) Cutri et al. (2014); 5) Evans et al. (2009); 6) this work.

Langlois et al. 2014) with and without coronagraph mask in the broad-band $J$ filter of SPHERE $\left(\lambda_{\mathrm{c}}=1.245 \mu \mathrm{m}\right)$. Both sets were divided in polarimetric cycles where each cycle contains four datacubes, one per half-wave plate (HWP) position angle (at $0^{\circ}, 22.5^{\circ}, 45^{\circ}$, and $67.5^{\circ}$, measured on sky east from north). The complete observation sequence amounted to $1.9 \mathrm{~h}$ on-source at airmass ranging from 1.7 to 1.8 , and standard calibration files (darks and flat fields) were provided by the ESO observatory.

The coronagraphic observations were taken with an apodized pupil Lyot coronagraph with a focal mask of $\sim 0$ '.09 in radius (Carbillet et al. 2011). Centre calibration frames with four satellite spots produced by a waffle pattern applied to the deformable mirror were taken at the beginning and at the end of the coronagraphic sequence to determine the position of the star behind the mask. In total, the coronagraphic observations amounted to $1.6 \mathrm{~h}$ on-source, with a median seeing of $00^{\prime \prime} \cdot 74 \pm 00^{\prime \prime} .12$ and coherence time of $5 \pm 1 \mathrm{~ms}$. The non-coronagraphic observations amounted to $0.3 \mathrm{~h}$ on-source under better weather conditions, with a median seeing of $00^{\prime \prime} 49 \pm 00^{\prime \prime} 06$ and coherence time of $8 \pm 1 \mathrm{~ms}$. We used detector integration times (DITs) of $64 \mathrm{~s}$ and $2 \mathrm{~s}$ for the coronagraphic and non-coronagraphic observations, respectively. A summary of the observations is given in Table 3 .

The HWP projects two simultaneous images with orthogonal polarization states over different regions on the detector. Subtracting these two images when the HWP is at $0^{\circ}\left(45^{\circ}\right)$ yields the Stokes parameter $Q^{+}\left(Q^{-}\right)$. Repeating this process 
Table 3. SPHERE observation log.

\begin{tabular}{cccccc}
\hline \hline Dataset & $\begin{array}{c}\text { Seeing } \\
\left({ }^{\prime \prime}\right)\end{array}$ & $\begin{array}{c}\tau \\
(\mathrm{ms})\end{array}$ & $\begin{array}{c}\text { DIT } \\
(\mathrm{s})\end{array}$ & Cycles & $\begin{array}{c}\text { Int. time } \\
(\mathrm{s})\end{array}$ \\
\hline Coronagraphic & 0.74 & 5 & 64 & 11 & 5632 \\
Non-Coro. & 0.49 & 8 & 2 & 14 & 1120 \\
\hline
\end{tabular}

Notes. The columns list the dataset, median seeing, median $\tau$, DIT, polarization cycles, and total integration time.

for the $22.5^{\circ}\left(67.5^{\circ}\right)$ angles produces the Stokes $U^{+}\left(U^{-}\right)$images. The total intensity (Stokes $I$ ) is computed by adding (instead of subtracting) the two images delivered by the HWP. We used customized scripts to process the raw data following the high-contrast imaging polarimetry pipeline described by Canovas et al. (2011). First, each science frame was dark current subtracted and flat-field corrected. Bad pixels (hot and dead) were identified with a $\sigma$ clipping algorithm and masked out using the average of their surrounding good pixels. The two simultaneous images from each science frame were first aligned using a cross-correlation algorithm. After this we applied an algorithm that finds the alignment that minimizes the standard deviation of the difference between the two images by shifting each image in steps of 0.05 pixels (see also Canovas et al. 2015b). This process was applied to every science frame resulting in a datacube for each Stokes $Q^{ \pm}, U^{ \pm}$parameter. These images were combined using the double-difference method (Canovas et al. 2011), yielding the observed Stokes $Q$ and $U$ parameters. At this stage we correct for instrumental polarization and instrument-induced cross-talk with the Mueller matrix model of van Holstein et al. (2017) and de Boer et al. (in prep.). This model describes the complete telescope+instrument system and is based on measurements with SPHERE's internal calibration lamp and observations of an unpolarized standard star. In this correction method every measurement of Stokes $Q$ and $U$ is described as a linear combination of the Stokes $I-, Q-$, and $U$-images incident on the telescope. The incident $Q$ - and $U$-images were obtained by solving, using linear least-squares, the system of equations describing all measurements. We then derived the polarization angle $\left(P_{\theta}=0.5 \arctan (U / Q)\right)$, the polarized intensity $\left(P_{I}=\right.$ $\sqrt{Q^{2}+U^{2}}$ ), and the $Q_{\phi}$ and $U_{\phi}$ images (see Schmid et al. 2006; Avenhaus et al. 2014b), with an accuracy below $\sim 0.5^{\circ}$ in $P_{\theta}$. This method is advantageous as no assumptions are made about the polarization of the star and the angle of linear polarization of the disc, and it has already been benchmarked against other pipelines (Pohl et al. 2017).

While the polarized images probe the disc surface, the intensity images alone can be used to derive detection limits on possible companions. Our observations were taken in field-tracking mode and no comparison star was observed, so high contrast algorithms (e.g. principal component analysis, Soummer et al. 2012) cannot be applied. Instead, we applied two image filters to the individual exposures to remove noise contributions from broad spatial features and to isolate point sources. The first filter removes azimuthally symmetric features like the stellar halo, as described in Wahhaj et al. (2013). The second filter is similar to the first, but it removes running flux averages over 20 pixels along the radial direction (instead of azimuthal) with the star as centre (see Wahhaj et al. 2016). This removes radial PSF features like diffraction spikes. We then computed the noise level of the individual intensity images in concentric rings centred over the star location. A contrast curve was computed by determining the $5 \sigma$ detection level at each radii after stacking all the intensity images.

\section{Results}

\subsection{The star}

Owing to the spread in distances and stellar parameters found in the literature, here we review the stellar properties of DZ Cha using our observations.

\subsubsection{Extinction}

Previous studies have used the intrinsic colours for main sequence (MS) M0 stars (Kenyon \& Hartmann 1995) obtaining a total extinction of $A_{V}=0.9$ (Wahhaj et al. 2010; Murphy et al. 2013). As DZ Cha has not yet entered the MS stage here we used the intrinsic colours for M0 pre-main sequence stars from Pecaut \& Mamajek (2013). Those were subtracted from the observed $(J-H),\left(J-K_{\mathrm{s}}\right)$, and $\left(H-K_{\mathrm{s}}\right)$ to obtain different colour excesses. These colours are not affected by veiling or by disc emission (see Sects. 3.1.3 and 3.2). The $A_{V}$ was computed from each excess assuming an extinction parameter of $R_{V}=3.1$ and the Fitzpatrick (1999) reddening law including the NIR empirical corrections by Indebetouw et al. (2005). We find that $A_{V}$ remains roughly constant independently of the colour index used, with an average value of $A_{V}=0.4 \pm 0.1$. We then used this value of $A_{V}$ to de-redden the entire photometry, and through our work we consider an extinction uncertainty of $\delta A_{V}=0.1$.

\subsubsection{Stellar properties}

The temperature scale for M0 PMS stars derived by Pecaut \& Mamajek (2013) yields $T_{\text {eff }}=3770 \pm 30 \mathrm{~K}$. We then compared the observed de-reddened photometry to the Phoenix/NextGen (Hauschildt et al. 1999; Allard et al. 2012) grid of synthetic stellar models in this temperature range. We chose the family of models computed for solar abundances (Asplund et al. 2009), with solar metallicity and zero $\alpha$-element enhancement (as expected for young stars in the solar neighbourhood), and with surface gravity in the range $\log g=[3.5-4.5]$. To directly compare the models with the observations we first normalized the synthetic flux at $J$ band $\left(\lambda_{\mathrm{c}}=1.2 \mu \mathrm{m}\right)$ to the observed value. In this band the stellar photosphere still dominates over the disc emission while the extinction uncertainties are lower than at optical bands. Integrating the photosphere at all wavelengths yields a stellar luminosity of $L_{\star}=0.6 \pm 0.1 L_{\odot}$, where the distance uncertainty is the dominant source of error. Provided with the effective temperature and stellar luminosity, we then used three different evolutionary tracks (Baraffe et al. 1998; Hauschildt et al. 1999; Siess et al. 2000) to estimate the stellar mass $\left(M_{\star}=0.70 \pm 0.15 M_{\odot}\right)$, radius $\left(R_{\star}=1.7 \pm 0.2 R_{\odot}\right)$, and age $(2.2 \pm 0.8 \mathrm{Myr})$. The radius and stellar mass yield a surface gravity of $\log g=3.7 \pm 0.1 \mathrm{~cm} \mathrm{~s}^{-2}$. Covino et al. (1997) estimated the projected rotational velocity $v \sin i$ of several WTTS including DZ Cha, where $v$ is the rotational velocity at the stellar equator and $i$ is the stellar inclination angle. They applied two different methods ${ }^{5}$ to their high resolution spectra $(\lambda / \Delta \lambda \sim 20000)$, obtaining $v \sin i=18 \pm 5 \mathrm{~km} \mathrm{~s}^{-1}$ and $v \sin i=13 \pm 5 \mathrm{~km} \mathrm{~s}^{-1}$. The UVES observations discussed in this paper have a spectral resolution that is nearly two times higher, so they can be used to further constrain this value. To

\footnotetext{
5 Covino et al. (1997) explain that their cross-correlation method (which yields $v \sin i=18 \pm 5 \mathrm{~km} \mathrm{~s}^{-1}$ for DZ Cha) is more reliable for slow rotators. To the best of our knowledge, all the following studies of DZ Cha quote only this value, ignoring the alternative estimate of $13 \pm 5 \mathrm{~km} \mathrm{~s}^{-1}$.
} 
Table 4. Stellar properties.

\begin{tabular}{ccc}
\hline \hline Parameter & Symbol & Value \\
\hline Spectral type $^{a}$ & $\mathrm{SpT}$ & $\mathrm{M} 0 \mathrm{Ve}$ \\
Distance $^{b}$ & $d$ & $110 \pm 7 \mathrm{pc}$ \\
Visual extinction & $A_{V}$ & $0.4 \pm 0.1$ \\
Effective temperature & $T_{\mathrm{eff}}$ & $3770 \pm 30 \mathrm{~K}$ \\
Luminosity & $L_{\star}$ & $0.6 \pm 0.1 L_{\odot}$ \\
Radius & $R_{\star}$ & $1.7 \pm 0.1 R_{\odot}$ \\
Mass & $M_{\star}$ & $0.7 \pm 0.2 M_{\odot}$ \\
Surface gravity & $\log g$ & $3.7 \pm 0.1 \mathrm{~cm} \mathrm{~s}^{-2}$ \\
Age & & $2.2 \pm 0.8 \mathrm{Myr}^{-1}$ \\
Projected velocity & $v \sin i$ & $\sim 8 \mathrm{~km} \mathrm{~s}^{-1}$ \\
\hline
\end{tabular}

Notes. ${ }^{(a)}$ Spectral type from Torres et al. (2006); ${ }^{(b)}$ distance from Murphy et al. (2013).

do so, we first synthesized a high resolution spectrum from the Phoenix/NextGen family of models using the effective temperature, metallicity, and surface gravity previously derived. This spectrum is then reddened and re-sampled at the same wavelengths as the UVES spectra. We then used the PyAstronomy package $^{6}$ to apply different rotational profiles to the synthetic spectrum, including limb-darkening corrections (using a limbdarkening coefficient of $\epsilon=0.6$ following Bouvier et al. 1986). Visual inspection of the synthesized spectra shows that models with $v \sin i=18 \mathrm{~km} \mathrm{~s}^{-1}$ produce absorption lines that are too broad, while models with $v \sin i \leq 13 \mathrm{~km} \mathrm{~s}^{-1}$, and in particular $v \sin i \sim 8 \mathrm{~km} \mathrm{~s}^{-1}$ (i.e. the lowest value within error bars estimated by Covino et al. 1997), produce a better match to our observations.

Batalha et al. (1998) and Messina et al. (2011) derived rotational periods $(P)$ of 11.7 and 8.0 days, respectively, using multiple epochs of high precision photometry at optical wavelengths. Computing the period as $P=2 \pi R_{\star} / v$, using our derived values of $R_{\star}=1.7 R_{\odot}$, and assuming that the stellar inclination is the same as the disc $\left(43^{\circ} \pm 5^{\circ}\right.$, see Sect. 3.2.2), we derive $P=3.3 \pm 0.3$ days for $v \sin i=18 \mathrm{~km} \mathrm{~s}^{-1}$. This discrepancy with the period values determined from the photometry was previously noticed by Messina et al. (2011). In contrast, using $v \sin i=8 \mathrm{~km} \mathrm{~s}^{-1}$ results in $P_{\max }=7.4 \pm 0.7$ days, in better agreement with the photometric periodic variability. Therefore, we adopt $v \sin i \sim 8 \mathrm{~km} \mathrm{~s}^{-1}$ as a representative value. The stellar properties are summarized in Table 4.

\subsubsection{Emission and absorption lines}

The optical spectra contain a wealth of prominent emission lines including the Balmer series up to $\mathrm{H} 14$, a few iron lines, and faint but significant emission in the [OI] $6300 \AA$ and [S II] $4068 \AA$ forbidden lines (Fig. 1). Given their importance as accretion and/or stellar activity tracers, we list the equivalent widths for the $\mathrm{H} \alpha$ and $\mathrm{H} \beta$ lines, the $\mathrm{Ca} \mathrm{K}$ and $\mathrm{H}$ doublet, the $\mathrm{He} \mathrm{I} 5876 \AA^{7}$, and the [OI] and [S II] forbidden lines in Table 5. The normalized profiles for $\mathrm{H} \alpha$, He I, [OI], and [S II] are shown in Fig. 2. There is no significant variation in the equivalent width in any of the lines except in $\mathrm{H} \alpha$, which shows a decrement from night 2 to night 3. We also computed the full width at $10 \%$ of the $\mathrm{H} \alpha$ line, finding that $\mathrm{FW} 0.1(\mathrm{H} \alpha)=128 \pm 2 \mathrm{~km} \mathrm{~s}^{-1}$ during the three nights.

\footnotetext{
6 https://github.com/sczesla/PyAstronomy

7 This line is a blend of the triplet $\mathrm{He} \mathrm{I}^{3} \mathrm{D}$ transition.
}

Table 5. Equivalent widths (in $\AA$ ) of the most significant emission lines.

\begin{tabular}{cccc}
\hline \hline Line ID & $E W_{1}$ & $E W_{2}$ & $E W_{3}$ \\
\hline Ca II K & $25.90 \pm 3.00$ & $21.25 \pm 3.10$ & $23.30 \pm 2.50$ \\
Ca II H & $15.70 \pm 3.10$ & $12.40 \pm 3.00$ & $13.40 \pm 3.00$ \\
[SII] & $0.24 \pm 0.10$ & $0.30 \pm 0.10$ & $0.21 \pm 0.10$ \\
H $\beta$ & $1.80 \pm 0.30$ & $1.85 \pm 0.30$ & $1.53 \pm 0.30$ \\
$\mathrm{He} \mathrm{I}$ & $0.12 \pm 0.06$ & $0.14 \pm 0.06$ & $0.10 \pm 0.06$ \\
[OI] & $0.12 \pm 0.02$ & $0.11 \pm 0.02$ & $0.10 \pm 0.02$ \\
$\mathrm{H} \alpha$ & $2.96 \pm 0.20$ & $3.16 \pm 0.20$ & $2.42 \pm 0.20$ \\
\hline
\end{tabular}

Notes. The subscripts indicate the first (2012-01-09), second (2012-0224), and third (2012-03-07) nights of the UVES observations (Table 1). Error bars represent the $3 \sigma$ uncertainty.

We have not found calibrated relations in the literature for M0 stars with FW0.1 $(\mathrm{H} \alpha)<150 \mathrm{~km} \mathrm{~s}^{-1}$, and using the relations derived by Natta et al. (2004) would result in an upper limit of $\dot{M}<10^{-11} M_{\odot} \mathrm{yr}^{-1}$. However, low $\left(\dot{M}<10^{-10} M_{\odot} \mathrm{yr}^{-1}\right)$ accretion rates derived from the $\mathrm{H} \alpha$ line profile alone can be unreliable because of contamination by chromospheric activity (e.g. Ingleby et al. 2011, 2013; Manara et al. 2013). The $\mathrm{H} \alpha$ line has a centro-symmetric double-peaked profile, very similar (both in shape and in equivalent width) to the $\mathrm{H} \alpha$ profiles observed around gas-poor discs like AU Mic (Houdebine \& Doyle 1994). The [OI] line is blue-shifted on all nights, with peak values ranging from -3.5 to $-6.8 \mathrm{~km} \mathrm{~s}^{-1}$. Fitting a Gaussian profile to the $[\mathrm{OI}]$ line results in Gaussian centres located at $-2.9 \pm 0.3 \mathrm{~km} \mathrm{~s}^{-1}$ and full width at half maximum $F W H M \sim 46 \pm 4 \mathrm{~km} \mathrm{~s}^{-1}$. Using the optical photometry and the line equivalent width we obtain a line luminosity of $L_{[\mathrm{OI}]}=3.1 \times 10^{-6} L_{\odot}$ with a $20 \%$ uncertainty.

A comparison between the synthesized stellar photosphere and the UVES spectra shows that the observed absorption lines are shallower than the model at wavelengths $\lesssim 6500 \AA$, while model and observations are in excellent agreement at longer wavelengths (Fig. 3, left panel). To further quantify this effect, we measured equivalent widths of some selected lines. In Fig. 3 (right panel) the ratio of the equivalent widths of the model and observations for several absorption lines at different wavelengths are shown. It is clear that the observations and model are in agreement at wavelengths $\gtrsim 6500 \AA$, while the observations have smaller equivalent widths than the model at bluer wavelengths. This effect is independent of the broadening factor used to model the synthetic spectra, and at first sight this seems to be the signature of veiling created by accretion shocks observed in many CTTS (e.g. Basri \& Batalha 1990; Hartigan et al. 1991).

\subsubsection{Ultraviolet excess}

The SED of DZ Cha (Fig. 4, left panel) shows a prominent UV excess. If this emission is caused by magnetospheric accretion or magnetic activity, then it can be reproduced by a scaled black body as

$F_{\mathrm{UV}}=X \frac{R_{\star}^{2}}{d^{2}} \pi B_{v}\left(T_{\mathrm{BB}}\right)$

where $B_{v}(T)$ is the Planck function at the black-body temperature $T_{\mathrm{BB}}$, and $X$ is a scaling factor. Fitting the two GALEX photometric points including distance and extinction uncertainties to this function we find that a black body at $T_{\mathrm{BB}}=12000_{-2000}^{+3000} \mathrm{~K}$ can match the UV excess (grey dot-dashed line in Fig. 4, left panel). With a luminosity of $\lesssim 0.001 L_{\odot}$ this black body 


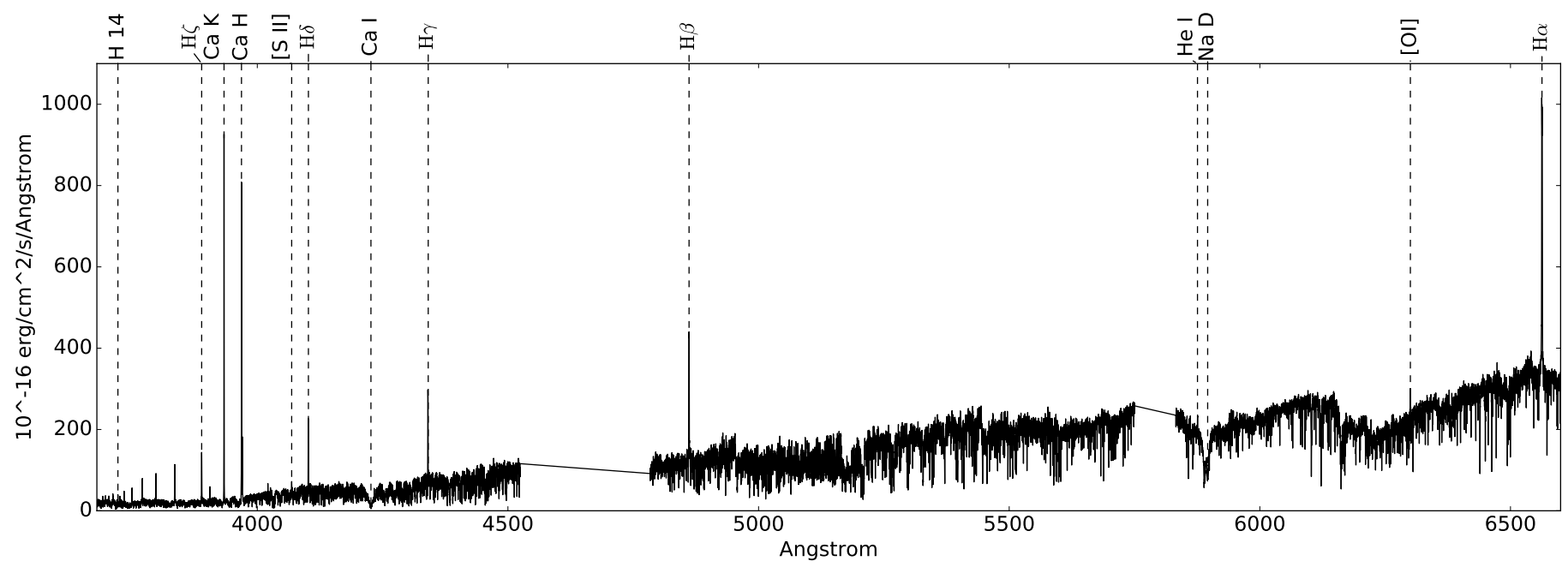

Fig. 1. Entire UVES spectrum of DZ Cha with selected emission lines highlighted. The hydrogen Balmer series is detected in emission up to the 14-2 (3721.946 ̊) transition. Only one spectrum is shown for clarity, as the three different spectra analysed here show almost no difference.

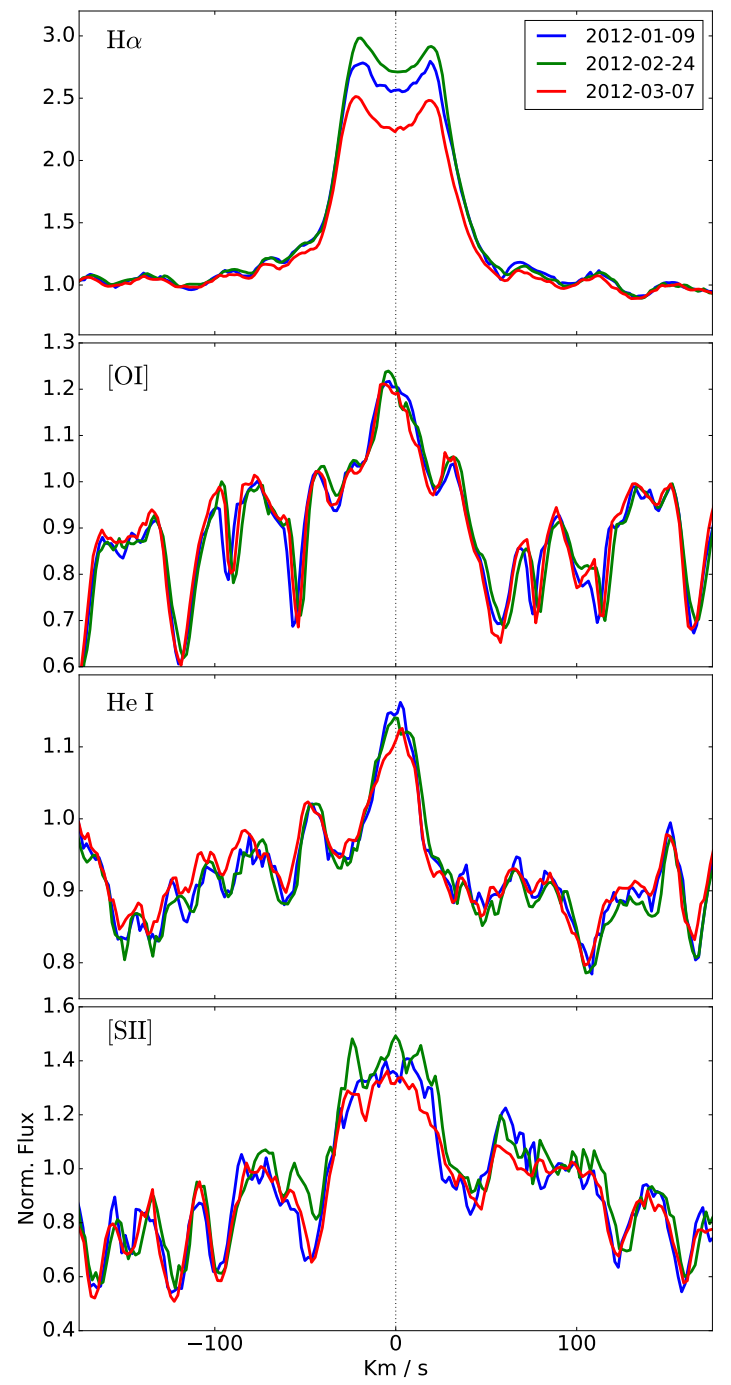

Fig. 2. Continuum normalized emission lines. The [OI] line is blueshifted in all nights, peaking at $\sim-5.1 \mathrm{~km} \mathrm{~s}^{-1}$.

is the dominant source of emission at UV wavelengths, but its contribution to the observed SED quickly becomes negligible, dropping below $<1 \%$ at wavelengths longer than $0.55 \mu \mathrm{m}$.
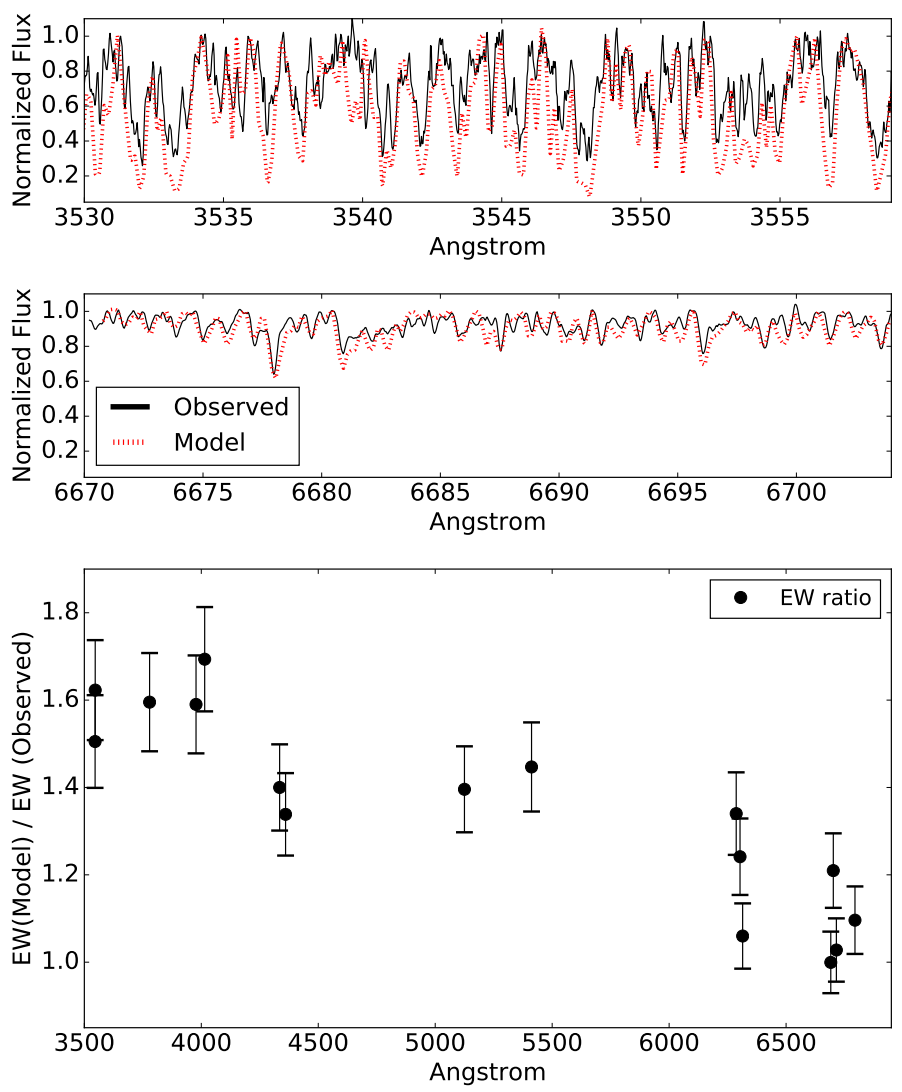

Fig. 3. Top: spectrum of DZ Cha in two different regions (black solid line) compared with the synthetic model (red dashed line). At red wavelengths the spectrum and model match each other and the lines have similar depths in both of them; at blue wavelengths the absorption lines of the spectrum are shallower than those in the model. Bottom: ratio of the equivalent widths of some selected lines across the spectrum plotted against the corresponding wavelengths. The ratios model/observations decrease as the wavelength increases. See text for details.

Assuming that all this flux is powered by accretion, then an independent estimate of the mass accretion rate can be obtained from the accretion luminosity as $L_{\text {acc }} \approx 0.8 G M_{\star} \dot{M} / R_{\star}$ (Gullbring et al. 1998). For DZ Cha, this yields to $\dot{M}<8.6 \times$ $10^{-11} M_{\odot} \mathrm{yr}^{-1}$, where the upper limit reflects that we have ignored the (significant) contribution from chromospheric activity. 
H. Canovas et al.: DZ Cha: a bona fide photoevaporating disc
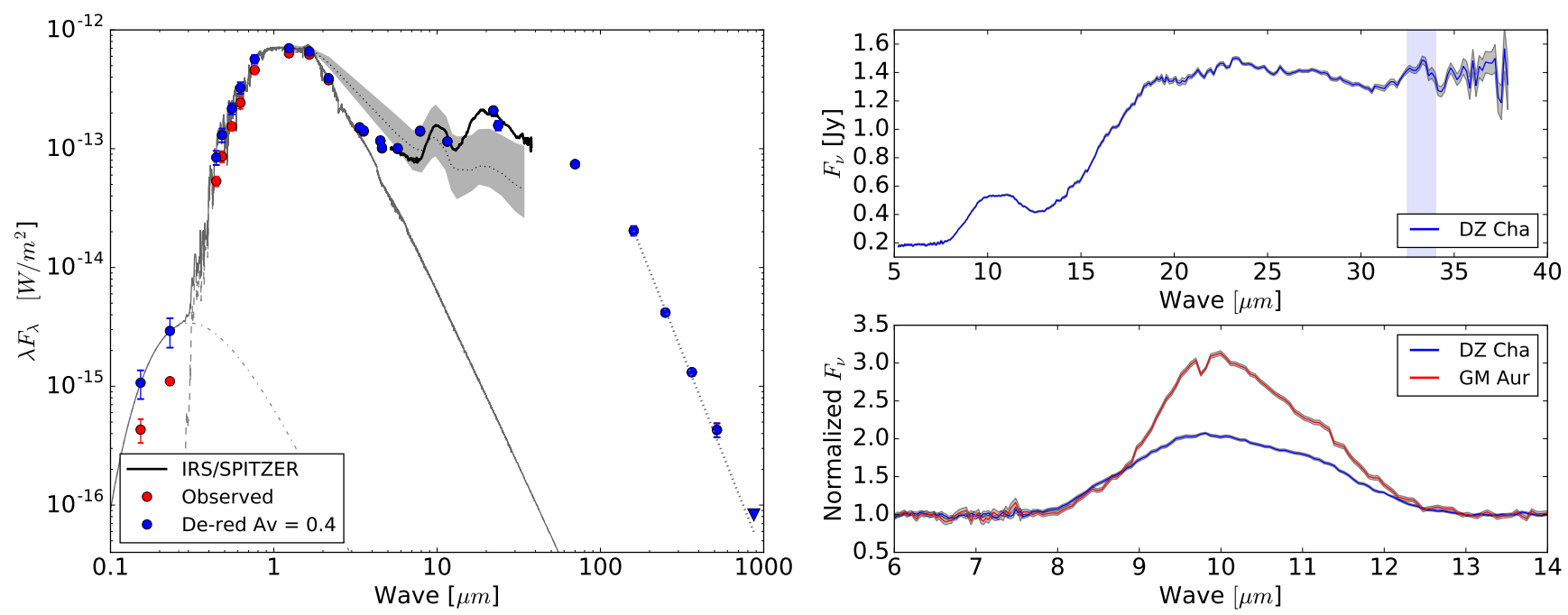

Fig. 4. Left: SED. The solid grey line shows the combination of a $12000 \mathrm{~K}$ black body (dot-dashed line) with the photosphere model (dashed line, see Sect. 3.1). The dotted black line and the grey shadowed region trace the median SED and upper/lower quartiles of Class II discs around K2-M5 stars in Taurus (Furlan et al. 2006). The dotted grey line shows the power-law fit $F_{v} \propto v^{\alpha}$, with $\alpha=2.5 \pm 0.1$ (see Sect. 3.2). Top right: IRS/Spitzer spectra. The light blue rectangle is centred around the $33.8 \mu \mathrm{m}$ crystalline silicate feature, and the grey shadowed region indicates the spectra uncertainties. Bottom right: de-redened and normalized $10 \mu \mathrm{m}$ profile of DZ Cha and GM Aur.

Considering all uncertainties and the narrow FW0.1(H $\alpha) \sim$ $130 \mathrm{~km} \mathrm{~s}^{-1}$ discussed above, we adopt an upper limit of $\dot{M}<$ $10^{-10} M_{\odot} \mathrm{yr}^{-1}$ for DZ Cha.

\subsection{Circumstellar disc}

\subsubsection{Infrared and sub-mm emission}

The SED and Spitzer/IRS spectra are plotted in Fig. 4 (left panel), along with the $J$-band normalized photosphere model described in the previous section. In the near-IR the SED is essentially photospheric at wavelengths shorter than $3.3 \mu \mathrm{m}$. Compared to the median emission of the K2-M5 stars with Class II SEDs (i.e. bright discs with strong thermal emission, most of them CTTS) observed in Taurus (Furlan et al. 2006), DZ Cha shows a clear deficit in flux up to $\sim 6 \mu \mathrm{m}$. This is the characteristic signature of the transitional discs (Strom et al. 1989). As already noted by these authors, this probably indicates dust-depleted central cavities of discs in the process of inside-out dispersal. The red IRAC colours [3.6]-[4.5] $=0.53$ and $[4.5]-[5.8]=0.59$ indicate that this cavity is not entirely devoid of dust (Hartmann et al. 2005). Beyond $6 \mu \mathrm{m}$ the SED shows robust thermal emission, with a prominent flux increment reaching the maximum at $\lambda \sim 20 \mu \mathrm{m}$ (corresponding to $\sim 145 \mathrm{~K}$ ). This near- and mid-infrared SED shape is similar to that of GM Aur, where the moderate NIR excess is explained by a small amount of hot dust and the steep rising in the continuum at IRS wavelengths indicates an inner wall directly exposed to the stellar radiation at temperature $T_{\text {wall }}=130 \mathrm{~K}$ (Calvet et al. 2005). Assuming that the dust grains in the wall re-emit the received energy from the star as a grey black body, a crude estimate of the wall location in DZ Cha can be derived from $d_{\text {wall }}=\left(R_{\star} T_{\text {eff }}\right)^{2} /\left(2 \sqrt{\epsilon} T_{\text {wall }}^{2}\right)$, where $\epsilon$ is the dust emissivity ( $\epsilon=1$ for a black body). For dust grains $\epsilon$ is very uncertain and depends on the grain properties. Using $T_{\text {wall }}=145 \mathrm{~K}$ and $\epsilon=[0.1-0.9]$ results in $d_{\text {wall }}=3-8$ au. At long wavelengths $(\lambda \geq 150 \mu \mathrm{m})$ the disc is expected to become more and more optically thin (e.g. Woitke et al. 2016) and its emission can be approximated as a black body radiating in the Rayleigh-Jeans regime, with $F_{v} \propto v^{\alpha}$. Fitting the observed $F_{v}$ fluxes at these wavelengths to a power law yields $\alpha=2.5 \pm 0.1$ (see the dotted line in the left panel of Fig. 4). This corresponds to a dust opacity index $\beta=\alpha-2=0.5$, much lower than the ISM $\beta \sim 1.7$ (Finkbeiner et al. 1999; Li \& Draine 2001). This difference indicates emission from large, probably $\mathrm{mm}$-sized and above, dust grains (Draine 2006; Testi et al. 2014). Assuming that the continuum emission at $870 \mu \mathrm{m}$ is optically thin and a representative average dust temperature the total dust mass can be estimated as

$$
M_{\text {dust }}=\frac{F_{v} d^{2}}{\kappa_{v} B_{v}\left(\left\langle T_{\text {dust }}\right\rangle\right)}
$$

(Hildebrand 1983), where $F_{v}$ is our $3 \sigma$ upper limit at frequency $v=345 \mathrm{GHz}(870 \mu \mathrm{m}), d$ is the distance to $\mathrm{DZ}$ Cha $(d=110 \mathrm{pc}), \kappa_{v}$ is the dust opacity, and $B_{v}\left(\left\langle T_{\text {dust }}\right\rangle\right)$ is the Planck function for a black body emitting at the average dust temperature, which is estimated following Andrews et al. (2013) as $\left\langle T_{\text {dust }}\right\rangle=25\left(L_{\star} / L_{\odot}\right)^{0.25}=22 \mathrm{~K}$. We assume that the dust opacity is a power law in frequency $\kappa_{v} \propto v^{\beta}$ normalized to $0.1 \mathrm{~cm}^{2} \mathrm{~g}^{-1}$ at $1000 \mathrm{GHz}$ for a gas-to-dust mass ratio of 100:1 (Beckwith et al. 1990). Using the previously derived opacity index of $\beta=0.5$ results in $\kappa_{345 \mathrm{GHz}}=2.8 \mathrm{~cm}^{2} \mathrm{~g}^{-1}$, which in turn yields a dust mass upper limit $M_{\text {dust }}<3 M_{\text {Earth }}$. Using a higher opacity index of e.g. $\beta=1.0$ results in an even lower upper limit $\left(M_{\text {dust }}<2.5 M_{\text {Earth }}\right)$, so we are confident about the disc being mostly optically thin at long wavelengths.

The IRS spectrum, shown in detail in Fig. 4 (right upper panel), shows a broad emission feature at $9.7 \mu \mathrm{m}$ typical of amorphous grains composed by silicates. Furthermore, at $\sim 33.8 \mu \mathrm{m}$, there is clearly a feature at the wavelength associated with crystalline forsterite (Koike et al. 2003). For comparison, we have normalized the continuum around the $10 \mu \mathrm{m}$ region for DZ Cha and for the protoplanetary disc GM Aur (Fig. 4, right bottom panel). In GM Aur, the shape of this feature indicates submicron sized (pristine) ISM grains (Sargent et al. 2006). In DZ Cha, the $10 \mu \mathrm{m}$ feature points to dust that is more evolved than that of GM Aur: the red shoulder, typical of larger ( $\gtrsim 2.0$ micron grains) is more pronounced, and the peak-to-continuum ratio is 2.07 , 


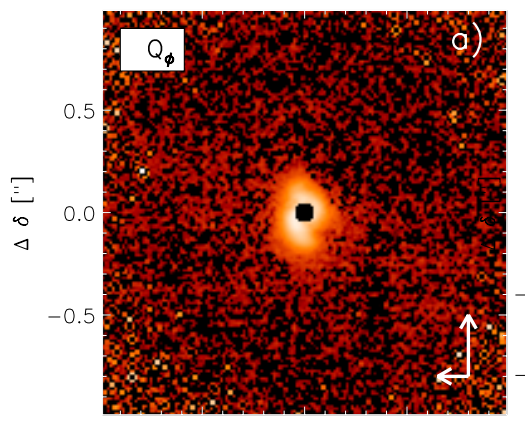

$\Delta \alpha\left[{ }^{\prime \prime}\right]$

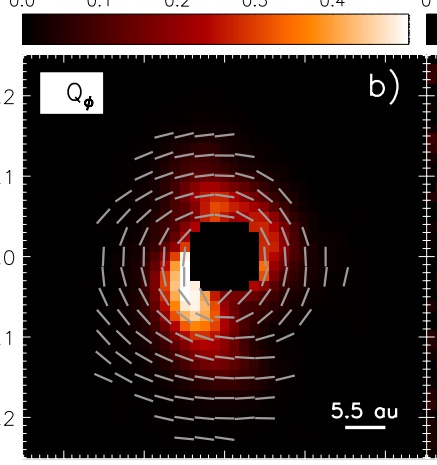

$\Delta \alpha[1]$
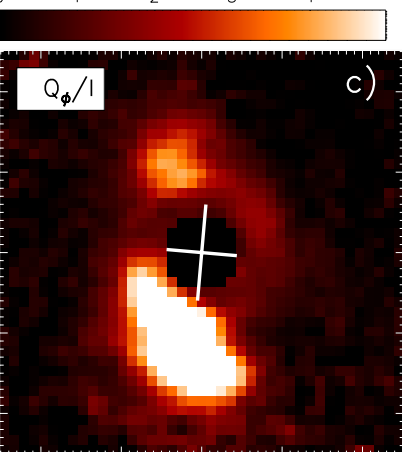

c)

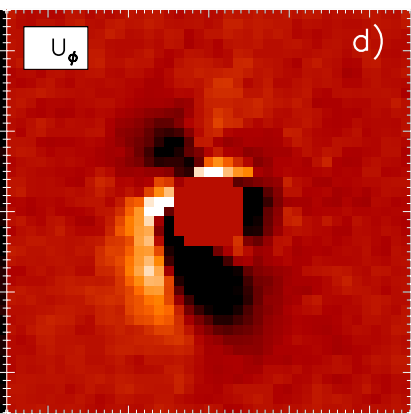

Fig. 5. Panel $a$ : $Q_{\phi}$ image of DZ Cha in logarithmic scale. The rest of the panels are shown in linear scale and the dynamical range of each image has been adjusted to better show the disc structure. Panel $b$ : Zoom-in of $a$ ) with the polarization angle $P_{\theta}$ plotted as grey vectors. Panel $c$ : Ratio of $Q_{\phi}$ over the total intensity $(I)$. The central cross indicates the projected major and minor disc axis. Panel $d$ : $U_{\phi}$ image normalized to the maximum value of $Q_{\phi}$. The positive/negative pattern is characteristic of multiple scattering events. In all panels the innermost region $(r<0$ '”04) dominated by speckle noise has been masked out; north is up and east is left.

while it is 3.13 for GM Aur. This peak/continuum ratio is shown to decrease as the dust grains grow (van Boekel et al. 2003). It is thus clear that dust processing has taken place in the disc of DZ Cha, as also indicated by the slope of the SED in the farinfrared and sub-mm ranges. There is no evident emission from the [Ne II] $12.8 \mu \mathrm{m}$ fine structure line. We have fit the continuum around the line to a second-order polynomial and subtract it to the spectra, finding a tentative $(\lesssim 2 \sigma)$ peak in emission at the line central wavelength. Given its low significance we give the $3 \sigma$ upper detection limit, which yields a line luminosity of $L_{\text {[NeII] }}<6.4 \times 10^{28} \mathrm{erg} \mathrm{s}^{-1}\left(<1.7 \times 10^{-5} L_{\odot}\right)$.

\subsubsection{The disc in polarized light}

Our images, which are sensitive to the small ( $\mu \mathrm{m}$-sized) dust grains that populate the disc upper layer, reveal a complex circumstellar environment. Polarized emission is detected in both the coronagraphic and non-coronagraphic observations. The coronagraphic images are blurrier than the unmasked ones, probably because of the different weather conditions that translated into a better AO performance in the unmasked dataset. The average point spread function (PSF) of the unmasked observations is almost diffraction limited with a FWHM of $\lesssim 00^{\prime \prime} 05$, and in this dataset polarized emission features are detected well inside the region masked out by the focal mask. Therefore, we focus our analysis on the unmasked observations (Fig. 5). The polarization angle $P_{\theta}$ is distributed in a nearly azimuthally symmetric pattern consistent with scattering by dust on a disc surface. The images show two elongated structures reminiscent of spiral arms in an $m=2$ rotational symmetry, with the northern (fainter) one extending from azimuthal angles $\left[270^{\circ}-20^{\circ}\right]$ (east of north), and the southern (brighter) one covering azimuthal angles $\left[100^{\circ}-190^{\circ}\right]$ (see central panels in Fig. 5). The $U_{\phi}$ image (panel d) shows a positive and negative pattern with a maximum value of $\sim 20 \%$ of the $Q_{\phi}$ image (peak to peak), and on average its amplitude is within $\sim 10 \%$ of $Q_{\phi}$. This is consistent with multiple scattering events in an inclined $\left(i \geq 40^{\circ}\right)$ disc (Canovas et al. 2015a). To highlight the disc morphology we applied different unsharp masks to the $Q_{\phi}$ images as in Garufi et al. (2016). The spiral-like features appear in every filter applied, showing in all cases a very similar morphology (Fig. 6). We also highlight two features (labelled "a" and "c") that seem spatially connected to

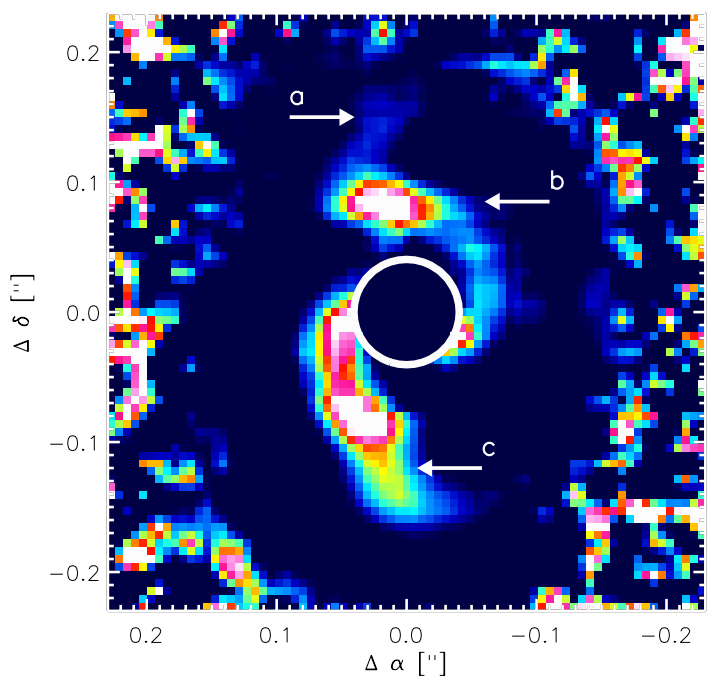

Fig. 6. Unsharped $Q_{\phi}$ image plotted in a hard stretch and colour scale. The inner $r<0$ '.04 region (white circle) is masked out. The arrows indicate different features discussed in the text.

the outer end of the spirals , and a bright clump (feature "b") in the northern spiral. Hints of these features are observed in the $Q_{\phi} / I$ panel in Fig. 5.

A first estimate of the disc structure can be obtained assuming that the disc is centro-symmetric and geometrically flat. We begin by smoothing the $Q_{\phi}$ image with a 2 px Gaussian kernel to reduce the impact of the bright and isolated features. We then fit isophotal ellipses to three different surface brightness ranges excluding the central $\left(r<00^{\prime \prime} 04\right)$ and outer $\left(r>00^{\prime \prime} 15\right)$ disc regions that are dominated by noise. This procedure was repeated using Gaussian kernels of increasing width (up to $10 \mathrm{px}$ ). This way we derive a position angle of $\mathrm{PA}=176^{\circ} \pm 7^{\circ}$ and an inclination of $i=43^{\circ} \pm 5^{\circ}$, where the quoted errors correspond to the standard deviation $(1 \sigma)$ of the different fits. We note that caution must be taken with our result as the disc is certainly not symmetric and probably flared (see Sect. 4.2).

The radial (polarized) brightness profile was measured by computing the mean in a 3 px slit ( $\sim 1$ resolution element) along the disc major axis (Fig. 7). The uncertainties at each position are 


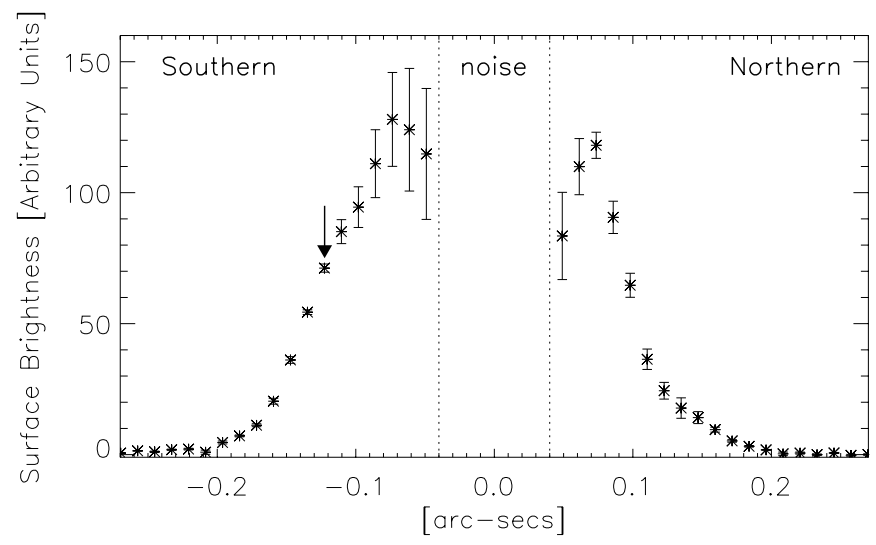

Fig. 7. Radial profile along the disc major axis. The southern and northern side peak at $\sim 0^{\prime \prime} 07 \pm 00^{\prime \prime} 01$. The arrow shows the radius where the brightness profile changes in slope along the southern side.

defined as the standard deviation divided by the square root of the slit width. The two semi-major axes peak at $00^{\prime \prime} 07 \pm 0$ '”01 and show an abrupt decrement in emission towards the star. This is the typical signature of a disc inner cavity. The semi-major axes are not symmetric, and overall the southern axis is brighter than the northern counterpart. The southern side is detected above $3 \sigma$ up to $0^{\prime \prime} .23 \pm 00^{\prime \prime} 01(\sim 25 \mathrm{au})$ from the star, while the northern side is detected up to $0^{\prime \prime} \cdot 18 \pm 00^{\prime \prime} \cdot 01(\sim 20 \mathrm{au})$. Neither of the two sides can be fitted by a single power law $\propto r^{\alpha}$, and the southern side shows a pronounced change in brightness at $0{ }^{\prime \prime} 12 \pm 00^{\prime \prime} .01$ $(\sim 13 \mathrm{au})$.

The dilution effect of the PSF can create a fake small central hole in polarized light (Avenhaus et al. 2014a). This artificial cavity depends on the PSF properties and, for example, a fake inner hole at 0 '.05 can be observed in diffraction limited observations at $K \mathrm{~s}$ band. To test whether the observed emission decrement near the star is an artefact we ran a set of simple toy models using the radiative transfer code MCFOST (Pinte et al. 2009). We created a continuous disc model (down to 0.1 au from the star) and a set of discs sharply truncated at an inner radius ranging from 5 to $9 \mathrm{au}$. We used a central star with the same properties as DZ Cha, a standard power law $\Sigma(r) \propto r^{-1}$ to describe the disc surface density distribution, a disc inclination of $43^{\circ}$, and a distance of $110 \mathrm{pc}$. Other disc properties such as grain composition or scale height have no impact for the purposes of this test. The modelled Stokes $Q$ and $U$ images were convolved with the average observed PSF, and synthetic $Q_{\phi}$ images were obtained from those. We then computed the cuts along the projected major axis as we did with the observations. Comparing the synthetic cuts with the observed one we found that the decrement in polarized flux is not an artefact but a real inner cavity of $7 \pm 0.5$ au in radius. This cavity size is in agreement with the inner wall location derived from our analysis of the SED shape (see Sect. 3.2.1).

\subsubsection{Companion detection limits}

The average $5 \sigma$ detection limits derived from our intensity images are shown in Fig. 8. Beyond 0'!2 (22 au projected) the contrast drops below $8 \mathrm{mag}$, reaching a roughly constant value of $\Delta m_{J} \sim 9.5 \mathrm{mag}$ at separations larger than $00^{\prime \prime} 4$. Using the distance and $J$-band magnitude of DZ Cha $\left(m_{J}=9.5\right)$, our detection limits imply that we should detect objects with absolute magnitude at $J$ band $M_{J} \lesssim 12.3$ at separations larger than 0 '!2. We used the AMES/Dusty models for brown dwarfs and giant

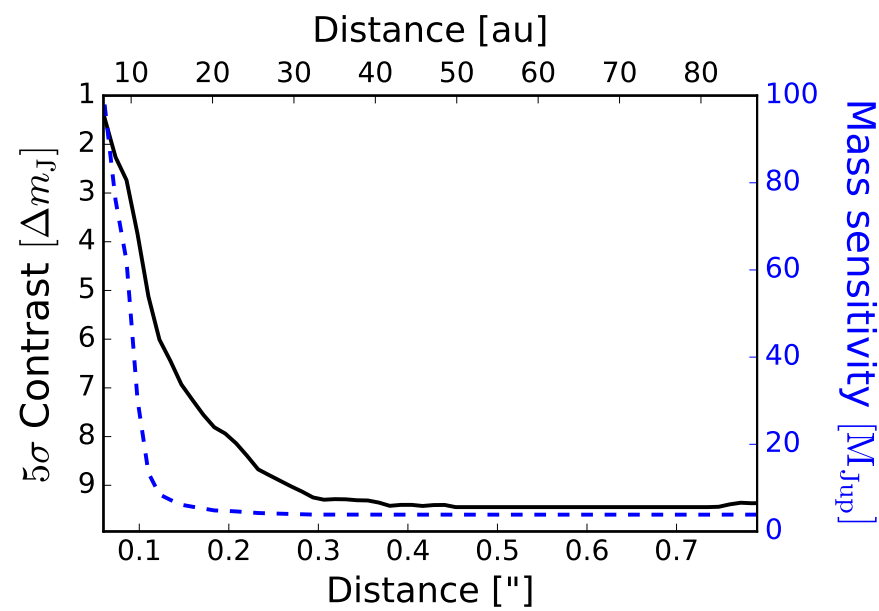

Fig. 8. Limits derived from the $J$-band intensity images. The solid black line traces the $5 \sigma$ contrast limit with its scale given on the $y$-left axis. The dashed blue line shows the mass sensitivity limits derived using AMES/Dusty models (Allard et al. 2001), with its corresponding scale on the $y$-right axis.

planets atmospheres (Allard et al. 2001) to derive mass sensitivity limits from our detection limits. The predicted temperature (and therefore brightness) depends on the assumed age, so for this exercise we use an age of 2.2 Myr (i.e. as if the companion were coeval with the central star). The mass sensitivity limits derived in this way are shown in Fig. 8. Giant planets with masses above $5 M_{\text {Jup }}$ orbiting beyond 22 au (projected) could be detected in our observations, although we note that the uncertainties are probably very high given the sensitivity of the planet brightness to the initial conditions of planet formation (Mordasini 2013) and that the disc emission may mask the planet signal. Our observations rule out stellar companions $\left(M_{\star}>80 M_{\text {Jup }}\right)$ down to 0 '.07 (projected $\sim 8 \mathrm{au}$ ) and equal mass companions down to 0 '”05 (projected $\sim 5 \mathrm{au}$ ).

Close-in stellar companions can be detected by combining several epochs of high resolution spectra as significant radial velocity variations will appear depending on the mass ratio and separation of the binary. Elliott et al. (2014) used this method to identify spectroscopic companions in a large sample including DZ Cha. They do not find evidence for binarity in DZ Cha using the same UVES dataset discussed here. Their result is consistent, within error bars, with previous measurements covering a time baseline of $\sim 20$ yr (Covino et al. 1997; Gregorio-Hetem et al. 1992; Torres et al. 2006). To further test whether the various radial velocity measurements of DZ Cha show statistically significant radial velocity variations we performed a $\chi^{2}$ test. As a null hypothesis we assumed that the average of the radial velocity measurements is a good representation of the constant radial velocity of DZ Cha. Using the $\chi^{2}$ probability function we find that the available observations do not provide any evidence for radial velocity variations. Combining the six independent measurements found in the literature results in an average radial velocity of $13.9 \pm 0.5 \mathrm{~km} \mathrm{~s}^{-1}$ ( $1 \sigma$ error computed including the individual uncertainties). In a binary system the radial velocity semi-amplitude $\left(K=v_{\mathrm{r}, \max }-v_{\mathrm{r}, \min }\right)$ and the semi-major axis (a) of the orbit are related via the Kepler laws. If the orbit is circular then $K=\sqrt{G} m_{2} \sin i\left(m_{1}+m_{2}\right)^{-0.5} a^{-0.5}$. Assuming that the orbit is coplanar with the disc $\left(i=43^{\circ}\right)$, and using a primary mass of $m_{1}=0.7 M_{\odot}$ and a $3 \sigma$ detection limit of $1.5 \mathrm{~km} \mathrm{~s}^{-1}$, the current measurements suggest that companions above $0.2 M_{\odot}$ at all separations are unlikely. 


\section{Discussion}

\subsection{Accretion or chromospheric emission?}

Many of the observed emission lines require temperatures well above the temperature of the stellar photosphere. In particular, the He I $5876 \AA$ line only shows up in emission when the temperature exceeds 8000 K (e.g. Saar et al. 1997; Beristain et al. 2001, and references therein). Furthermore, the spectra also show veiling signatures (Sect. 3.1.3). In TTS two mechanisms can produce the high energies needed to create these features: accretion and magnetic activity.

In young stars, the Balmer lines, the $\mathrm{He} \mathrm{I}$, and the $\mathrm{Ca}$ II lines can originate from a very hot inner wind or from an accretion shock (Batalha et al. 1996; Muzerolle et al. 1998; Beristain et al. 2001; Stempels \& Piskunov 2002; Kwan \& Fischer 2011), while veiling is usually considered a signature of accretion activity, (e.g. Gullbring et al. 1998; Hartmann et al. 1998). However, the narrow $\mathrm{H} \alpha$ line profile argues against accretion in DZ Cha. The $\mathrm{H} \alpha$ line is known to be variable in TT stars (with the FW0.1 $(\mathrm{H} \alpha$ ) having a typical dispersion of $0.65 \mathrm{dex}$; Nguyen et al. 2009), but our three different spectra and the observations from Wahhaj et al. (2010) show a nearly constant FW0.1 $(\mathrm{H} \alpha)<130 \mathrm{~km} \mathrm{~s}^{-1}$. Similarly, combining our three spectra with observations at five different epochs from the literature we find that the equivalent width consistently remains around $E W(\mathrm{H} \alpha)=4 \pm 2 \AA$ in timescales from months to years, except during two flaring events (Gregorio-Hetem et al. 1992; Alcala et al. 1995; Guenther \& Emerson 1997; Tripicchio et al. 2000; Riaz et al. 2006; Torres et al. 2006). According to Martin et al. (1998), M0-M2 stars with $E W(\mathrm{H} \alpha)>10 \AA$ are accreting. White \& Basri (2003) find that CTTS have FW0.1 $(\mathrm{H} \alpha)>270 \mathrm{~km} \mathrm{~s}^{-1}$, independently of their spectral type, and this limit drops to $200 \mathrm{~km} \mathrm{~s}^{-1}$ for substellar objects (Jayawardhana et al. 2003). Barrado y Navascués \& Martín (2003) propose an empirical criterion based on the saturation limit traced by $\log [L(\mathrm{H} \alpha) / L(\mathrm{bol})]=-3.3$. In this scenario, M0 CTTS have $E W(\mathrm{H} \alpha)>8 \AA$ (with small variations depending on the observed star forming region, see their Fig. 4). In short the $\mathrm{H} \alpha$ line profile indicates no accretion in DZ Cha, as this object is not even in the boundary between WTTS and CTTS in any of these widely used empirical criteria. Furthermore, the He I $5876 \AA$ line profile also argues against accretion in DZ Cha. Accreting objects usually have larger equivalent widths in the He I line (EW(He I)) than non-accreting ones. The EW(He I) in DZ Cha is $\sim 30 \times$ lower than the $\mathrm{EW}(\mathrm{He}$ I) of the accreting K7M2 PMS stars studied by Alcalá et al. (2014), and is $\sim 3 \times$ lower than the EW(He I) of the non-accreting K7-M2 PMS stars in the sample studied by Manara et al. (2013).

An alternative and plausible explanation for the UV excess, veiling, and emission lines is chromospheric activity. Ultraviolet excesses caused by chromospheric emission alone have already been observed in a number of WTTS and magnetically active M dwarf stars (Houdebine et al. 1996; Ingleby et al. 2013). DZ Cha is magnetically active as it shows flaring events (Guenther \& Emerson 1997; Tripicchio et al. 2000). Therefore, the UV excess observed in DZ Cha could be caused by just chromospheric emission, and this excess may account for the observed veiling signatures. Similarly, high transitions of the Balmer series and several emission lines observed in DZ Cha (e.g. $\mathrm{He} \mathrm{I}, \mathrm{H} \alpha, \mathrm{Ca}$ ) are also observed in WTTS due to chromospheric emission alone (Manara et al. 2013). Finally, it could be argued that short episodes of enhanced accretion cause outbursts that mimic the observational signatures of the stellar flares. However, young outbursting objects such as FU Ori and EX Ori stars have average accretion rates in quiescence of $\sim 10^{-7} M_{\odot} \mathrm{yr}^{-1}$, and their outbursts increase the stellar flux at optical wavelengths by a few magnitudes over at least several months (Audard et al. 2014, and references therein). This is clearly not the case in DZ Cha, so the two flaring events observed to date in this system indeed indicate intense magnetic activity.

Therefore, and taking into account the previous discussion about the $\mathrm{H} \alpha$ line and accretion variability, we conclude that magnetic activity, and not accretion, is the mechanism heating the stellar chromosphere and causing the observed UV excess, emission lines, and veiling.

\subsection{A complex and evolved disc}

Our observations show that DZ Cha has undergone significant evolution from its primordial stage, where dust grains in the circumstellar disc should be ISM-like and the inner disc should extend down to the dust sublimation limit $(<0.5$ au for TTS, Muzerolle et al. 2003). In DZ Cha, the IRS spectrum presents the signature of crystal silicates at $\sim 33.8 \mu \mathrm{m}$, while the shape of the $10 \mu \mathrm{m}$ feature indicates $\gtrsim 2.0 \mu \mathrm{m}$ dust grains in the optically thin upper layers of the disc. The spectral index $\beta \sim 0.5$ derived from the far-infrared and sub-mm SED probes the continuum emission from large, likely mm-sized, grains. Furthermore, the strong infrared excess indicates that the disc is optically thick at most infrared wavelengths. Our polarized images and in particular the brightness surface profile along the disc major axis show a dust cavity (Fig. 7). Taking into account the dilution effect of the PSF we find that this cavity is $\sim 7$ au in radius. An optically thin dust-depleted cavity is also evident from the transitional disc-like SED, which shows no excess below $3.3 \mu \mathrm{m}$ and has a prominent increment from 6 to $20 \mu \mathrm{m}$ characteristic of an inner wall directly exposed to the stellar radiation. The relatively small outer radius of $\sim 30$ au from our observations suggests a compact disc, but deep observations at longer wavelengths are needed to accurately constrain the disc size. Our upper mass limit from the $870 \mu \mathrm{m}$ photometry ( $<3 M_{\text {Earth }}$, Sect. 3.2.1) indicates a total mass $M_{\text {disc }}<0.95 M_{\text {Jup }}$ assuming a conservative gas-to-dust mass ratio of 100:1. Using our derived stellar mass $\left(M_{\star}=0.5 M_{\odot}\right.$, Table 4$)$, we obtain an upper limit for the stardisc dust mass ratio of $M_{\text {disc }} / M_{\star}<0.2 \%$.

The spiral features observed in the polarized images seem to be in an approximate $m=2$ rotational symmetry, hinting at an undetected companion. The theory shows that if this is the case, the observed morphology depends on several factors including disc properties (like the $\alpha$ viscosity parameter, scale height, and inclination), the star/companion ratio $q$, and the companion location (e.g. Dong et al. 2015; Juhász et al. 2015). Current models predict that when observed in NIR scattered light images, outward spirals (those launched beyond the companion) are more difficult to detect than inward spirals (Dong et al. 2015; Zhu et al. 2015; Dong \& Fung 2017). Independently of the companion mass, outward spirals are expected to have very small pitch angles, in contrast to the open arms predicted when the spiral is located between the companion and the star. Therefore, the bright and open spirals observed in DZ Cha suggest inward spirals launched by a companion orbiting in the outer disc. In our images the spirals are not detected beyond $\sim 20$ au, so we adopt this value as the minimum orbit of the hypothetic companion. The minimum mass of a companion driving a detectable spiral feature in NIR scattered light depends on the detailed properties of the disc, and for DZ Cha it might be around $\sim 0.1 M_{\text {Jup }}$ (Dong \& Fung 2017). The morphology and brightness of the $Q_{\phi}$ 
and $U_{\phi}$ images is roughly consistent with an inclined generic disc with inward spiral arms driven by a $0.5-50 M_{\text {Jup }}$ companion ( $q=0.003,0.1$; Dong et al. 2016). Our point-source detection limits suggest that such a companion should be $\lesssim 5 M_{\text {Jup }}$. Altogether we conclude that a companion with masses in the $0.1-5 M_{\text {Jup }}$ range driving inward spirals and orbiting at $r_{\text {orbit }}>$ 20 au might explain the observed morphology of DZ Cha.

The lack of accretion does not necessarily imply a gas-poor disc and, in fact, there is indirect and direct observational evidence of gas in DZ Cha. The infrared excess of DZ Cha is much stronger than the excesses found around gas-poor discs (e.g. Eiroa et al. 2013). The overall SED shape and disc fractional luminosity of DZ Cha are, however, very similar to those observed in the flared gas-rich discs around CTTS. Direct evidence of the presence of gas in the disc comes from our detection of the forbidden [OI] and [SII] emission lines in all three epochs. These lines are usually detected in CTTS, and their low velocity in our spectra indicate that they trace emission from warm gas in the disc surface (e.g. Hartigan et al. 1995; Natta et al. 2014).

\subsection{A bona fide photoevaporating disc}

Mechanisms like grain growth, viscous accretion, photoevaporation, and planet formation, are expected to play different roles in the disc evolution depending on the intrinsic properties of the star+disc system (see e.g. Espaillat et al. 2014). This is probably reflected on the diversity of SEDs observed in the WTTS population (e.g. Cieza et al. 2013). Below we discuss how the properties of DZ Cha argue against most disc evolution mechanisms except photoevaporation. We then show that the current observational evidence indicates that DZ Cha is most likely a bona fide photoevaporating disc at the inner stages of disc clearing.

In Sect. 3.2 we showed that grain growth is happening in DZ Cha. Grain growth alone can explain small $(<10 \mathrm{au})$ disc inner cavities (Birnstiel et al. 2012), but this process cannot explain the negligible accretion rate observed in DZ Cha across different epochs (see Sect. 4.1). Giant planets ( $\geq 1 M_{\text {Jup }}$ ) carve gaps and/or cavities in the dust and gas distribution of protoplanetary discs (Lubow et al. 1999; Pinilla et al. 2012). These planets can decrease the accretion rate onto the star by $10-20 \%$ (Lubow \& D'Angelo 2006). Considering the typical accretion rates of CTTS $\left(10^{-7}-10^{-9} M_{\odot} \mathrm{yr}^{-1}\right.$, Gullbring et al. 1998), the low upper limit for the accretion rate in DZ Cha cannot be accounted for by one giant planet alone. Multiple and vigorously accreting giant planets may reduce the stellar accretion rate to $\lesssim 10^{-10} M_{\odot} \mathrm{yr}^{-1}$, but then very large disc cavities should be created (Zhu et al. 2011). Therefore, planet-disc interactions cannot explain the observed properties of DZ Cha. Poorly ionized disc regions (dead-zones) can locally have low accretion rates creating gaps in the disc, but the stellar accretion rate is expected to remain insensitive to these dead-zones (Gammie 1996), and therefore this mechanism cannot explain negligible accretion rates. Finally, binary stellar companions can carve inner cavities of $\sim 2-3 a$ in radius in their circumbinary discs, where $a$ is the semi-major axis of the binary orbit (Artymowicz \& Lubow 1994). The impact of binarity in the accretion rate is not yet well quantified, but there are both theoretical and observational studies showing that the accretion flow continues via accretion streams through the dust cleared central cavity of circumbinary discs (Artymowicz \& Lubow 1996; Basri et al. 1997). Most importantly, none of the mechanisms enumerated above can explain the disc outflow evidenced by the detection of the forbidden $[\mathrm{SII}]$ and $[\mathrm{OI}]$ lines in the UVES spectra.
The shape and luminosities of the forbidden lines, and the stellar X-ray luminosity, can be used to directly compare model predictions with observations. In Sect. 3.2.1 we obtained $L_{[\mathrm{NeII}]}<6.4 \times 10^{28} \mathrm{erg} \mathrm{s}^{-1}\left(<1.7 \times 10^{-5} L_{\odot}\right)$. Taking into account previous observations (e.g. Lahuis et al. 2007; Pascucci \& Sterzik 2009), it seems plausible that the IRS spectra discussed here just do not have enough sensitivity to detect this line. To further explore this avenue we compared our observations with the X-ray and EUV photoevaporation models presented by Ercolano \& Owen (2010). Scaling the DZ Cha X-ray luminosity given by Malo et al. (2014) to 110 pc we obtain $\log \left(L_{\mathrm{X}}\right)=30.09 \mathrm{erg} \mathrm{s}^{-1}$. Ercolano \& Owen (2010) compute several line luminosities for stars with $X$-ray luminosities in the $\log L_{\mathrm{X}}=28.3-30.3 \mathrm{erg} \mathrm{s}^{-1}$ range. From these models we estimate a $\left[\mathrm{Ne}\right.$ II] luminosity of $L_{[\mathrm{NeII}]} \lesssim 3.2 \times 10^{-6} L_{\odot}$, i.e. about 5 times below the sensitivity limit of our IRS observations. The detection limit of $L_{[\mathrm{NeII}]}<1.7 \times 10^{-5} L_{\odot}$ is unfortunately too high to derive a meaningful estimate of the photoevaporative mass loss rate from this line (following e.g. Hollenbach \& Gorti 2009; Ercolano \& Owen 2010). Repeating the same exercise with the [O I] line $\left(L_{[\mathrm{OI}]} \sim 3.1 \times 10^{-6} L_{\odot}\right.$, Sect. 3.1.3) we find a good agreement between observations and models (with expected luminosities in the $L_{[\mathrm{OI}]}=1.9 \times 10^{-6}-1.2 \times 10^{-5}$ range for stellar $X$ rays luminosities of $\log L_{X}=29.3-30.3$ ). The line peak velocity is also in agreement with those models, which predict $v_{\text {peak }}=[-0.75,-14] \mathrm{km} \mathrm{s}^{-1}$ for discs with $\sim 8$ au cleared inner cavities inclined by $40^{\circ}-50^{\circ}$. The observed FWHM of $\sim 46 \pm 4 \mathrm{~km} \mathrm{~s}^{-1}$ is, however, $\sim 60 \%$ higher than the models' predictions. Mass loss rates in a photoevaporative wind depend on the energy of the stellar radiation. Using Eq. (9) in Owen et al. (2012) we obtain a mass loss rate due to X-ray photoevaporation of $\dot{M}_{\text {wind }} \sim 9.8 \times 10^{-9} M_{\odot} \mathrm{yr}^{-1}$, which is nearly 2 orders of magnitude higher than the mass accretion rate derived for DZ Cha (Sect. 3.1.4).

Photoevaporation alone gives an elegant explanation to most of the observed features in DZ Cha. As explained in Sect. 1, the stellar photons with energies in the X-ray-UV range can heat the gas in the disc surface by injecting the gas molecules with energy enough to overcome the disc gravitational potential (Clarke et al. 2001; Alexander et al. 2006b; Gorti \& Hollenbach 2009; Ercolano et al. 2009; Owen et al. 2012). The inner disc detaches from the outer disc at a critical radius once the mass loss rates due to photoevaporation equal or exceed the mass accretion rates. For a $0.5 M_{\odot}$ star like DZ Cha, the critical radius from EUV radiation is expected to be at $R_{\mathrm{C}} \sim 1$ au (Alexander et al. 2014, and references therein). When this happens the inner disc quickly drains in its local viscous timescale, the accretion onto the star stops, and the inner wall of the outer disc is directly irradiated by the central star. This inner wall then shifts outwards and the entire disc dissipates from the inside out in $\sim 10^{5} \mathrm{yr}$ (Alexander et al. 2006b, 2014). The multi-epoch observations discussed here indicate a very low accretion rate ( $\dot{M}<10^{-10} M_{\odot} \mathrm{yr}^{-1}$ ) on timescales from months to years. The SED and images indicate a cavity size of $\sim 7$ au in radius filled with a small amount of optically thin hot dust. This is consistent with the picture previously outlined: in DZ Cha the inner disc has probably already drained (explaining the negligible accretion rate) and the inner wall has moved outwards by up to $\sim 7 \mathrm{au}$. The broad and slightly blue-shifted [OI] $6300 \AA$ A emission is consistent with a warm flow of photoevaporated gas escaping from the disc surface (Ercolano \& Owen 2010, 2016). Finally, the low disc mass $\left(M_{\text {disc }}<0.9 M_{\text {Jup }}\right)$ is consistent with UVdominated photoevaporation, as these models predict that the cavity will open when most of the disc mass has already been 
accreted by the star (Alexander et al. 2006b), as opposed to the expected higher mass of discs with cavities carved out by giant planets or X-ray photoevaporating discs (Ercolano et al. 2009; Owen et al. 2012).

\section{Summary and conclusions}

In this work we analyse three epochs of high resolution optical spectroscopy with UVES, high contrast $J$-band imaging polarimetry with SPHERE, mid-infrared spectroscopy with IRS/Spitzer, and the overall SED from the UV up to the sub$\mathrm{mm}$ regime of DZ Cha. Comparing our results with previous observations we obtain nine epochs of high resolution spectroscopy encompassing time baselines from years to months. We find that $E W(\mathrm{H} \alpha)$ and $\mathrm{FW} 0.1(\mathrm{H} \alpha)$ of DZ Cha remain well below the WTTS/CTTS boundary, indicating that accretion has stopped or is below $\dot{M}<10^{-10} M_{\odot} \mathrm{yr}^{-1}$. Exceptions to this are two reported flaring events in which the $\mathrm{H} \alpha$ line was significantly broadened (Covino et al. 1997; Guenther \& Emerson 1997; Tripicchio et al. 2000). The UVES spectra analysed here show emission lines indicative of high temperatures (e.g. He I $5876 \AA$ ) and veiling-like effects. We show that given the lack of accretion in this system, and its important flaring events, an active chromosphere is most likely causing the observed veiling, emission lines, and UV excess. The analysis of the SED and IRS spectra reveals that the disc around DZ Cha shows evidence of grain growth, probably up to mm sizes and above, and signatures from crystal silicates. Using our APEX photometry we derive an upper dust mass limit of $M_{\text {dust }}<3 M_{\text {Earth }}$. Our polarized images reveal a $\sim 43^{\circ}$ inclined disc with two spiral-like features and a dust depleted cavity of $\sim 7 \mathrm{au}$, which is broadly consistent with the near- and mid-infrared shape of the SED. The spiral shape and polarized $Q_{\phi}$ and $U_{\phi}$ images match the model predictions of an inclined disc with spiral arms driven by planetary mass body exterior to the spirals.

Although grain growth is needed to explain the far-infrared SED slope, and planet-disc interactions can explain the observed spiral features, we argue that the overall observational characteristics of DZ Cha strongly suggest photoevaporation as the mechanism leading the evolution of DZ Cha. Most importantly, we show that its observed properties are consistent with a disc at the earlier stages of photoevaporation. At these stages the inner disc has already drained, accretion has stopped, and the inner wall of the outer disc is exposed to stellar radiation (e.g. Alexander et al. 2006a). DZ Cha is by no means the only bright disc showing a small accretion rate (e.g. Sicilia-Aguilar et al. 2006a,b; Cieza et al. 2008), and there are even objects that appear to switch between the CTTS and WTTS categories (Littlefair et al. 2004). For example, IM Lup and Sz 68 have low accretion rates, nearly at the WTTS boundary, and SEDs similar to that of a CTTS with continuous and massive discs (Pinte et al. 2008; Cieza et al. 2013). However, the combination of negligible accretion rates over the last $20 \mathrm{yr}$, the relatively small disc cavity, the forbidden emission lines, and the low disc mass, all in agreement with photoevaporation models, seem to be a unique feature of DZ Cha. We consider that DZ Cha is an ideal target to benchmark photoevaporation theories against direct observations.

Acknowledgements. The authors thank the referee, Dr. R. Alexander, for his useful comments and suggestions which helped to improve this manuscript. H.C. acknowledges useful discussions with J. Caballero and E. Covino. H.C., C.E., G.M., B.M., I.R., and E.V. are supported by Spanish grant AYA 2014-55840-P. G.M. acknowledges support from Spanish grant RyC-2011-07920. M.R.S. L.C., C.C., and A.H. acknowledge founding by the Millennium Science Initiative, Chilean Ministry of Economy, Nucleus RC130007. L.A.C. acknowledges support from CONICYT FONDECYT grant 1171246. C.C. acknowledges support from project CONICYT PAI/Concurso Nacional Insercion en la Academia, convocatoria 2015, Folio 79150049. This publication makes use of data products from 1) the AAVSO Photometric All Sky Survey (APASS), funded by the Robert Martin Ayers Sciences Fund and the National Science Foundation; 2) the Two Micron All Sky Survey, which is a joint project of the University of Massachusetts and the Infrared Processing and Analysis Center/California Institute of Technology, funded by the National Aeronautics and Space Administration and the National Science Foundation; and 3) the Wide-field Infrared Survey Explorer, which is a joint project of the University of California, Los Angeles, and the Jet Propulsion Laboratory/California Institute of Technology, funded by the National Aeronautics and Space Administration. This research has made use of 1) the services of the ESO Science Archive Facility; 2) the Spanish Virtual Observatory (http://svo.cab.inta-csic.es) supported by the Spanish MICINN/MINECO through grants AyA2008-02156, AyA2011-24052; 3 ) the NASA/IPAC Infrared Science Archive, which is operated by the Jet Propulsion Laboratory, California Institute of Technology, under contract with the National Aeronautics and Space Administration; 4) the VizieR catalogue access tool, CDS, Strasbourg, France; and 4) Astropy, a community-developed core Python package for Astronomy Astropy Collaboration 2013.

\section{References}

Alcala, J. M., Krautter, J., Schmitt, J. H. M. M., et al. 1995, A\&AS, 114, 109 Alcalá, J. M., Natta, A., Manara, C. F., et al. 2014, A\&A, 561, A2 Alexander, R. D., Clarke, C. J., \& Pringle, J. E. 2006a, MNRAS, 369, 216 Alexander, R. D., Clarke, C. J., \& Pringle, J. E. 2006b, MNRAS, 369, 229 Alexander, R., Pascucci, I., Andrews, S., Armitage, P., \& Cieza, L. 2014, Protostars and Planets VI, 475

Allard, F., Hauschildt, P. H., Alexander, D. R., Tamanai, A., \& Schweitzer, A. 2001, ApJ, 556, 357

Allard, F., Homeier, D., \& Freytag, B. 2012, Philos. Trans. Roy. Soc. London Ser. A, 370, 2765

Andrews, S. M., \& Williams, J. P. 2005, ApJ, 631, 1134

Andrews, S. M., Rosenfeld, K. A., Kraus, A. L., \& Wilner, D. J. 2013, ApJ, 771, 129

Ansdell, M., Williams, J. P., van der Marel, N., et al. 2016, ApJ, 828, 46

Artymowicz, P., \& Lubow, S. H. 1994, ApJ, 421, 651

Artymowicz, P., \& Lubow, S. H. 1996, ApJ, 467, L77

Asplund, M., Grevesse, N., Sauval, A. J., \& Scott, P. 2009, ARA\&A, 47, 481

Audard, M., Ábrahám, P., Dunham, M. M., et al. 2014, Protostars and Planets VI, 387

Avenhaus, H., Quanz, S. P., Meyer, M. R., et al. 2014a, ApJ, 790, 56

Avenhaus, H., Quanz, S. P., Schmid, H. M., et al. 2014b, ApJ, 781, 87

Baraffe, I., Chabrier, G., Allard, F., \& Hauschildt, P. H. 1998, A\&A, 337, 403

Barrado y Navascués, D., \& Martín, E. L. 2003, AJ, 126, 2997

Basri, G., \& Batalha, C. 1990, ApJ, 363, 654

Basri, G., Johns-Krull, C. M., \& Mathieu, R. D. 1997, AJ, 114, 781

Batalha, C. C., Stout-Batalha, N. M., Basri, G., \& Terra, M. A. O. 1996, ApJS, 103,211

Batalha, C. C., Quast, G. R., Torres, C. A. O., et al. 1998, A\&AS, 128, 561

Beckwith, S. V. W., Sargent, A. I., Chini, R. S., \& Guesten, R. 1990, AJ, 99, 924 Beristain, G., Edwards, S., \& Kwan, J. 2001, ApJ, 551, 1037

Bertout, C. 1989, ARA\&A, 27, 351

Beuzit, J.-L., Feldt, M., Dohlen, K., et al. 2008, in SPIE Conf. Ser., 7014, 18

Bianchi, L., Herald, J., Efremova, B., et al. 2011, Ap\&SS, 335, 161

Birnstiel, T., Andrews, S. M., \& Ercolano, B. 2012, A\&A, 544, A79

Bouvier, J., Bertout, C., Benz, W., \& Mayor, M. 1986, A\&A, 165, 110

Calvet, N., D’Alessio, P., Watson, D. M., et al. 2005, ApJ, 630, L185

Canovas, H., Ménard, F., de Boer, J., et al. 2015a, A\&A, 582, L7

Canovas, H., Perez, S., Dougados, C., et al. 2015b, A\&A, 578, L1

Canovas, H., Rodenhuis, M., Jeffers, S. V., Min, M., \& Keller, C. U. 2011, A\&A, 531, A102

Carbillet, M., Bendjoya, P., Abe, L., et al. 2011, Exp. Astron., 30, 39

Cieza, L., Padgett, D. L., Stapelfeldt, K. R., et al. 2007, ApJ, 667, 308

Cieza, L. A., Swift, J. J., Mathews, G. S., \& Williams, J. P. 2008, ApJ, 686, L115

Cieza, L. A., Olofsson, J., Harvey, P. M., et al. 2013, ApJ, 762, 100

Clarke, C. J., Gendrin, A., \& Sotomayor, M. 2001, MNRAS, 328, 485

Covino, E., Alcala, J. M., Allain, S., et al. 1997, A\&A, 328, 187

Cutri, R. M., Skrutskie, M. F., van Dyk, S., et al. 2003, VizieR Online Data Catalog: II $/ 246$

Cutri, R. M., et al. 2014, VizieR Online Data Catalog: II/328

Dohlen, K., Langlois, M., Saisse, M., et al. 2008, in SPIE Conf. Ser., 7014, 3

Dong, R., \& Fung, J. 2017, ApJ, 835, 38

Dong, R., Zhu, Z., Rafikov, R. R., \& Stone, J. M. 2015, ApJ, 809, L5

Dong, R., Fung, J., \& Chiang, E. 2016, ApJ, 826, 75 
Draine, B. T. 2006, ApJ, 636, 1114

Duvert, G., Guilloteau, S., Ménard, F., Simon, M., \& Dutrey, A. 2000, A\&A 355,165

Eiroa, C., Marshall, J. P., Mora, A., et al. 2013, A\&A, 555, A11

Elliott, P., Bayo, A., Melo, C. H. F., et al. 2014, A\&A, 568, A26

Ercolano, B., \& Owen, J. E. 2010, MNRAS, 406, 1553

Ercolano, B., \& Owen, J. E. 2016, MNRAS, 460, 3472

Ercolano, B., Clarke, C. J., \& Drake, J. J. 2009, ApJ, 699, 1639

Espaillat, C., Muzerolle, J., Najita, J., et al. 2014, Protostars and Planets VI, 497

Evans, II, N. J., Allen, L. E., Blake, G. A., et al. 2003, PASP, 115, 965

Evans, II, N. J., Dunham, M. M., Jørgensen, J. K., et al. 2009, ApJS, 181, 321

Finkbeiner, D. P., Davis, M., \& Schlegel, D. J. 1999, ApJ, 524, 867

Fitzpatrick, E. L. 1999, PASP, 111, 63

Font, A. S., McCarthy, I. G., Johnstone, D., \& Ballantyne, D. R. 2004, ApJ, 607, 890

Furlan, E., Hartmann, L., Calvet, N., et al. 2006, ApJS, 165, 568

Gammie, C. F. 1996, ApJ, 457, 355

Garufi, A., Quanz, S. P., Schmid, H. M., et al. 2016, A\&A, 588, A8

Gorti, U., \& Hollenbach, D. 2009, ApJ, 690, 1539

Gregorio-Hetem, J., Lepine, J. R. D., Quast, G. R., Torres, C. A. O., \& de La Reza, R. 1992, AJ, 103, 549

Guenther, E. W., \& Emerson, J. P. 1997, A\&A, 321, 803

Gullbring, E., Hartmann, L., Briceño, C., \& Calvet, N. 1998, ApJ, 492, 323

Güsten, R., Nyman, L. A., Schilke, P., et al. 2006, A\&A, 454, L13

Hardy, A., Caceres, C., Schreiber, M. R., et al. 2015, A\&A, 583, A66

Hartigan, P., Kenyon, S. J., Hartmann, L., et al. 1991, ApJ, 382, 617

Hartigan, P., Edwards, S., \& Ghandour, L. 1995, ApJ, 452, 736

Hartmann, L., Calvet, N., Gullbring, E., \& D'Alessio, P. 1998, ApJ, 495, 385

Hartmann, L., Megeath, S. T., Allen, L., et al. 2005, ApJ, 629, 881

Hauschildt, P. H., Allard, F., \& Baron, E. 1999, ApJ, 512, 377

Henden, A. A., Templeton, M., Terrell, D., et al. 2016, VizieR Online Data Catalog : II/336

Hildebrand, R. H. 1983, QJRAS, 24, 267

Hollenbach, D., \& Gorti, U. 2009, ApJ, 703, 1203

Hollenbach, D., Johnstone, D., Lizano, S., \& Shu, F. 1994, ApJ, 428, 654

Houdebine, E. R., \& Doyle, J. G. 1994, A\&A, 289, 185

Houdebine, E. R., Mathioudakis, M., Doyle, J. G., \& Foing, B. H. 1996, A\&A, 305,209

Howard, C. D., Sandell, G., Vacca, W. D., et al. 2013, ApJ, 776, 21

Indebetouw, R., Mathis, J. S., Babler, B. L., et al. 2005, ApJ, 619, 931

Ingleby, L., Calvet, N., Bergin, E., et al. 2011, ApJ, 743, 105

Ingleby, L., Calvet, N., Herczeg, G., et al. 2013, ApJ, 767, 112

Jayawardhana, R., Mohanty, S., \& Basri, G. 2003, ApJ, 592, 282

Joy, A. H. 1945, ApJ, 102, 168

Juhász, A., Benisty, M., Pohl, A., et al. 2015, MNRAS, 451, 1147

Kenyon, S. J., \& Hartmann, L. 1995, ApJS, 101, 117

Koike, C., Chihara, H., Tsuchiyama, A., et al. 2003, A\&A, 399, 1101

Kovács, A. 2008, in Millimeter and Submillimeter Detectors and Instrumentation for Astronomy IV, Proc. SPIE, 7020, 70201

Kwan, J., \& Fischer, W. 2011, MNRAS, 411, 2383

Lahuis, F., van Dishoeck, E. F., Blake, G. A., et al. 2007, ApJ, 665, 492

Langlois, M., Dohlen, K., Vigan, A., et al. 2014, in Ground-based and Airborne Instrumentation for Astronomy V, Proc. SPIE, 9147, 91471

Li, A., \& Draine, B. T. 2001, ApJ, 554, 778

Littlefair, S. P., Naylor, T., Harries, T. J., Retter, A., \& O'Toole, S. 2004 MNRAS, 347, 937

Lopez Martí, B., Jimenez Esteban, F., Bayo, A., et al. 2013, A\&A, 551, A46

Lubow, S. H., \& D’Angelo, G. 2006, ApJ, 641, 526

Lubow, S. H., Seibert, M., \& Artymowicz, P. 1999, ApJ, 526, 1001

Luhman, K. L., Allen, L. E., Allen, P. R., et al. 2008, ApJ, 675, 1375

Macías, E., Anglada, G., Osorio, M., et al. 2016, ApJ, 829, 1

Malo, L., Doyon, R., Lafrenière, D., et al. 2013, ApJ, 762, 88

Malo, L., Artigau, É., Doyon, R., et al. 2014, ApJ, 788, 81
Manara, C. F., Testi, L., Rigliaco, E., et al. 2013, A\&A, 551, A107

Martin, E. L., Montmerle, T., Gregorio-Hetem, J., \& Casanova, S. 1998, MNRAS, 300, 733

Messina, S., Desidera, S., Lanzafame, A. C., Turatto, M., \& Guinan, E. F. 2011, A\&A, 532, A10

Mordasini, C. 2013, A\&A, 558, A113

Murphy, S. J., Lawson, W. A., \& Bessell, M. S. 2013, MNRAS, 435, 1325

Muzerolle, J., Calvet, N., \& Hartmann, L. 1998, ApJ, 492, 743

Muzerolle, J., Calvet, N., Hartmann, L., \& D'Alessio, P. 2003, ApJ, 597, L149

Natta, A., Testi, L., Muzerolle, J., et al. 2004, A\&A, 424, 603

Natta, A., Testi, L., Alcalá, J. M., et al. 2014, A\&A, 569, A5

Nguyen, D. C., Scholz, A., van Kerkwijk, M. H., Jayawardhana, R., \& Brandeker, A. 2009, ApJ, 694, L153

Owen, J. E., Clarke, C. J., \& Ercolano, B. 2012, MNRAS, 422, 1880

Padgett, D. L., Cieza, L., Stapelfeldt, K. R., et al. 2006, ApJ, 645, 1283

Pascucci, I., \& Sterzik, M. 2009, ApJ, 702, 724

Pecaut, M. J., \& Mamajek, E. E. 2013, ApJS, 208, 9

Pinilla, P., Benisty, M., \& Birnstiel, T. 2012, A\&A, 545, A81

Pinte, C., Padgett, D. L., Ménard, F., et al. 2008, A\&A, 489, 633

Pinte, C., Harries, T. J., Min, M., et al. 2009, A\&A, 498, 967

Pohl, A., Sissa, E., Langlois, M., et al. 2017, A\&A, 605, A34

Quanz, S. P., Amara, A., Meyer, M. R., et al. 2015, ApJ, 807, 64

Riaz, B., Gizis, J. E., \& Harvin, J. 2006, AJ, 132, 866

Rigliaco, E., Pascucci, I., Gorti, U., Edwards, S., \& Hollenbach, D. 2013, ApJ, 772,60

Riviere-Marichalar, P., Pinte, C., Barrado, D., et al. 2013, A\&A, 555, A67

Saar, S. H., Huovelin, J., Osten, R. A., \& Shcherbakov, A. G. 1997, A\&A, 326, 741

Sacco, G. G., Flaccomio, E., Pascucci, I., et al. 2012, ApJ, 747, 142

Sallum, S., Follette, K. B., Eisner, J. A., et al. 2015, Nature, 527, 342

Sargent, B., Forrest, W. J., D’Alessio, P., et al. 2006, ApJ, 645, 395

Schmid, H. M., Joos, F., \& Tschan, D. 2006, A\&A, 452, 657

Sicilia-Aguilar, A., Hartmann, L., Calvet, N., et al. 2006a, ApJ, 638, 897

Sicilia-Aguilar, A., Hartmann, L. W., Fürész, G., et al. 2006b, AJ, 132, 2135

Siess, L., Dufour, E., \& Forestini, M. 2000, A\&A, 358, 593

Siringo, G., Kreysa, E., Kovács, A., et al. 2009, A\&A, 497, 945

Skrutskie, M. F., Cutri, R. M., Stiening, R., et al. 2006, AJ, 131, 1163

Soummer, R., Pueyo, L., \& Larkin, J. 2012, ApJ, 755, L28

Stempels, H. C., \& Piskunov, N. 2002, A\&A, 391, 595

Strom, K. M., Strom, S. E., Edwards, S., Cabrit, S., \& Skrutskie, M. F. 1989, AJ, 97, 1451

Testi, L., Birnstiel, T., Ricci, L., et al. 2014, Protostars and Planets VI, 339

Torres, C. A. O., Quast, G. R., da Silva, L., et al. 2006, A\&A, 460, 695

Torres, C. A. O., Quast, G. R., Melo, C. H. F., \& Sterzik, M. F. 2008, Young Nearby Loose Associations, ed. B. Reipurth, 757

Tripicchio, A., Alcala, J. M., Covino, E., \& D’Antona, F. 2000, Mem. Soc. Astron. It., 71, 1021

van Boekel, R., Waters, L. B. F. M., Dominik, C., et al. 2003, A\&A, 400, L21

van Holstein, R. G., Snik, F., Girard, J. H., et al. 2017, Proc. SPIE, 10400, 1040015

Wahhaj, Z., Cieza, L., Koerner, D. W., et al. 2010, ApJ, 724, 835

Wahhaj, Z., Liu, M. C., Biller, B. A., et al. 2013, ApJ, 779, 80

Wahhaj, Z., Milli, J., Kennedy, G., et al. 2016, A\&A, 596, L4

Walter, F. M., Brown, A., Mathieu, R. D., Myers, P. C., \& Vrba, F. J. 1988, AJ, 96, 297

White, R. J., \& Basri, G. 2003, ApJ, 582, 1109

Winn, J. N., \& Fabrycky, D. C. 2015, ARA\&A, 53, 409

Woitke, P., Min, M., Pinte, C., et al. 2016, A\&A, 586, A103

Wolk, S. J., \& Walter, F. M. 1996, AJ, 111, 2066

Zhu, Z., Nelson, R. P., Hartmann, L., Espaillat, C., \& Calvet, N. 2011, ApJ, 729, 47

Zhu, Z., Dong, R., Stone, J. M., \& Rafikov, R. R. 2015, ApJ, 813, 88 